\title{
MODELO TRÓFICO DEL ECOSISTEMA ROCOSO LITORAL DE LA ISLA DE EL HIERRO, ISLAS CANARIAS
}

\author{
José Carlos Mendoza* y José Carlos Hernández \\ Universidad de La Laguna
}

\begin{abstract}
Resumen
Presentamos un modelo de balance de masas realizado mediante el programa Ecopath with Ecosim para caracterizar la estructura trófica y los principales flujos de energía del ecosistema sublitoral rocoso de la isla de El Hierro. El modelo se compone de 22 grupos funcionales, que representan las especies más características de las comunidades bentónicas de las islas Canarias. Los indicadores ecológicos y parámetros obtenidos en el modelo de balance de masas caracterizaron el ecosistema como un sistema bentónico en desarrollo, alejado de la madurez y con un bajo reciclaje de la materia, dominado en términos de biomasa por los niveles tróficos bajos e intermedios. Se identificó a los grupos de peces ictiófagos, de niveles tróficos altos, como las especies clave del ecosistema. Este modelo puede ser utilizado para la exploración de escenarios temporales que analicen el efecto de diferentes tipos de perturbaciones sobre los grupos clave que contribuyen a la resiliencia del ecosistema. Por lo que se trata de una herramienta de gran valor para los gestores de los recursos marinos de El Hierro, quienes pueden prever los posibles efectos ecosistémicos de nuevas medidas de gestión pesquera.
\end{abstract}

Palabras Clave: modelo trófico, pesquerías, isla de El Hierro, ecosistemas costeros.

\section{A TROPHIC MODEL OF EL HIERRO SHALLOW ROCKY BOTTOM ECOSYSTEM, CANARY ISLANDS}

\begin{abstract}
We provide a mass-balance model which has been constructed using Ecopath with Ecosim software to characterize the trophic structure and the main energy flows of the sublittoral rocky-bottom ecosystem of El Hierro island. The model is composed by 22 trophic groups, that represent the most characteristic species of the benthic communities of the Canary Islands. The ecological indicators and parameters resulted from the mass balance model characterized the ecosystem as an undeveloped system, far from the maturity status, with a low recycling index and dominated by mid-low trophic levels in terms of biomass. Macroinvertebrate feeders and piscivorous fish groups were identified as keystone species of the ecosystem. This model can be used for exploring different temporal scenarios to a nalyse the effect of different type of perturbations on keystone groups that contribute to ecosystem resilience. In this sense, this model is a very valuable tool for the managers of El Hierro marine resources who can foresee the possible ecosystem effects of new fisheries policies.
\end{abstract} Keywords: trophic model, fisheries, El Hierro Island, Coastal Ecosystems. 


\section{INTRODUCCION}

La isla de El Hierro $\left(27^{\circ} 45^{\prime} \mathrm{N}, 18^{\circ} 00^{\prime} \mathrm{O}\right)$ es un enclave biológico de carácter volcánico y subtropical, situado en el archipiélago de las islas Canarias. Las aguas que rodean la isla son de carácter oceánico y oligotrófico (De León y Braun 1973; Braun 1980; Hernández 2016), sin embargo, existen unos recursos marinos diversos (Bortone et al. 1991) y que presentan un buen estado de conservación, sobre todo en la reserva marina localizada en el Mar de Las Calmas y sus alrededores (Hernández et al., 2008). En este área, están bien representados los hábitats costeros de macroalgas más conspicuos del archipiélago canario, los fondos de Lobophoras (Sangil et al., 2011). Además, y debido a la abundancia de recursos marinos de interés pesquero que presenta la isla de El Hierro, se ha establecido una flota artesanal que centra su actividad pesquera en las aguas circundantes (De la Cruz-Modino 2011).

Durante las últimas décadas, el interés por el estudio del impacto de las actividades humanas en los hábitats y comunidades marinas está desarrollando la aplicación de un enfoque ecosistémico (Cowan et al. 2012). Este enfoque holístico, útil para entender las dinámicas de los ecosistemas marinos y sus poblaciones, ha convertido la modelización ecológica en una herramienta fundamental para su análisis (Christensen y Walters 2004; Plagányi 2007). En este sentido, los modelos tróficos permiten caracterizar la estructura y funcionamiento de los ecosistemas marinos, considerando las interacciones multiespecíficas que ocurren dentro de los mismos. De esta forma, podemos construir una herramienta ecológica que nos proporciona información estratégica en la gestión y toma de decisiones sobre los ecosistemas marinos que nos permita proyectar en el tiempo los resultados obtenidos para simular escenarios futuros.

Uno de los enfoques que permite caracterizar los ecosistemas marinos es el modelo trófico de equilibrio de masas «Ecopath with Ecosim» (EwE) (Polovina 1984; Christensen y Pauly 1992). La aplicación de los modelos EwE (en su módulo temporal y espacial) también permite realizar modelizaciones ecológicas para evaluar tendencias temporales como los efectos de la pesca, la implementación de áreas marinas protegidas o simular escenarios de cambio climático (Pauly et al. 2000; Christensen y Walters 2004, 2011). En el módulo estacionario (Ecopath), en el que se centra este trabajo, los análisis cuantitativos de flujos de materia y energía están relacionadas con indicadores ecológicos que nos informan de las propiedades del ecosistema como la resiliencia y el estado de madurez del mismo (Odum 1969). El análisis de estos indicadores ecológicos puede ser de gran utilidad para contribuir al conocimiento de la capacidad de recuperación de los ecosistemas frente al impacto de las actividades humanas (Ulanowicz 1986).

En los ecosistemas costeros explotados como es el caso de la isla de El Hierro, los modelos Ecopath pueden contribuir a describir la estructura, complejidad y

* Ecología de Comunidades Marinas y Cambio Climático. Dpto. Biología Animal, Edafología y Geología (U.D. Ciencias Marinas). Facultad de Ciencias (Biología). Universidad de La Laguna, Tenerife, Canary Islands, Spain. Autor para la correspondencia: jcmendoza85@gmail.com. 
funcionamiento de las comunidades que lo componen como una herramienta para la conservación. Nuestro estudio se ha centrado en el ecosistema marino de la isla de El Hierro, para construir un modelo trófico Ecopath. De esta forma pretendemos caracterizar los fondos rocosos someros de El Hierro que nos sirva de base para establecer predicciones en base a diferentes medidas de conservación o manejos pesqueros. En las islas Canarias, la aplicación de modelos $E w E$ es todavía muy escasa. Hasta el momento, se ha desarrollado un modelo trófico en la laguna de Maspalomas (Moreno y Teresa 1998) y un modelo pesquero (Couce-Montero et al. 2015) en la isla de Gran Canaria. Sin embargo, no existe un modelo trófico que ponga de manifiesto la caracterización y funcionamiento de los fondos marinos en la isla de El Hierro. Para la región del Atlántico-oriental, se han realizado otros modelos ecológicos como en la costa atlántica de Marruecos (Stanford et al. 2001), en el archipiélago de Azores, Portugal (Guénette y Morato 2001), en el archipiélago de Cabo Verde (Stobberup et al. 2004) y el golfo de Cádiz, España (Torres et al. 2013).

Por todo ello, con el fin de obtener un análisis trófico del ecosistema costero de El Hierro, se construyó un modelo de equilibrio de masas para: (1) describir la estructura trófica y los principales flujos de energía; (2) analizar los indicadores ecológicos del modelo para describir las características del ecosistema desde la perspectiva de la Teoría Ecológica (Lindeman 1942; Odum 1969; Ulanowicz 1986, 1995), evaluando la resiliencia y madurez del mismo; y (3) comparar los atributos tróficos e indicadores ecológicos con los demás modelos parametrizados de la región Atlántico-oriental, concretamente con los de Torres et al. 2013 y Couce-Montero et al. 2015.

\section{MATERIAL Y MÉTODOS}

\section{1. ÁrEA y PERÍODO DE ESTUdIO}

El área representativa de los ecosistemas marinos someros (hasta los 40 metros de profundidad) de El Hierro comprende la región del Mar de Las Calmas, situada en la vertiente Suroeste de la Isla. Concretamente, el área modelizada (7.46 $\mathrm{km}^{2}$ ) queda integrada en los límites de la Reserva Marina de interés pesquero Punta Restinga-Mar de Las Calmas (RM-RMC), donde opera principalmente la flota pesquera artesanal (figura 1). El área de estudio, de aguas calmadas y pobres en nutrientes, se caracteriza por presentar unos fondos rocosos muy escarpados donde predominan las comunidades de macroalgas y una gran biodiversidad de especies ícticas (Brito et al. 2001; Falcón et al. 1996; Falcón 2016; Tuya et al. 2004).

Además, esta región de la Isla cuenta con un plan de seguimiento científico y la disponibilidad de datos de abundancias de especies y descargas de pesca, desde que se creó la Reserva Marina en 1996, hasta la actualidad. El monitoreo de la abundancia de algas, peces e invertebrados marinos, mediante el seguimiento in situ de buceo autónomo se lleva a cabo desde 1997. Para la representación del modelo estacionario se eligió el período de tiempo del año 2003, por contar con la mejor calidad y abundancia de datos locales, tanto en las estimaciones de biomasa como en el registro de capturas de pesca por la flota artesanal de La Restinga. Todos los 
datos incluidos en el modelo se han obtenido de la bibliografía, que ha sido especificada, en detalle, en el anexo 1.

\section{2. Есоратн with Ecosim (EwE)}

Para la construcción del modelo de equilibrio de masas de la isla de El Hierro, se utilizó el módulo Ecopath (Polovina 1984; Christensen y Pauly 1992) del programa informático EwE (Ecopath with Ecosim), version 6.6 (Christensen y Walters, 2004; Christensen et al. 2008; Heymans et al. 2016). En términos generales, el módulo Ecopath describe de forma cuantitativa las interacciones tróficas y la transferencia de energía que hay en el modelo, en un estado de equilibrio en sus grupos tróficos y para un intervalo de tiempo establecido.

El modelo asume el equilibrio de masas mediante una serie de ecuaciones lineales simultáneas, una para cada especie o grupo trófico del sistema. La primera ecuación describe la producción en cada grupo funcional (i) de la siguiente manera:

$$
\begin{gathered}
\text { Producción }(\mathrm{i})=\text { Depredación sobre }(\mathrm{i})+\text { Mortalidad por pesca }(\mathrm{i})+\text { Otras causas } \\
\text { de mortalidad (i) + Biomasa acumulada (i) + Migración neta }
\end{gathered}
$$

En términos matemáticos, la primera ecuación se puede expresar como:

$$
\text { Bi . (P/B)i . EEi - } \sum \text { Bj . (Q/B)j . DCij - Yi - Ei - BAi = } 0
$$

donde Bi es la biomasa del grupo funcional (i); (P/B)i es la producción del grupo funcional (i) por unidad de biomasa y es equivalente a la mortalidad total $(Z)$ en un estado estacionario (Allen 1971); EE es la eficiencia ecotrófica de (i); (Q/B)i es el consumo de (i) por unidad de biomasa; DCij es la proporción de (i) en la dieta del depredador (j) en unidades de volumen o peso; Yi es el total de capturas de (i) por unidad de tiempo y área por la flota pesquera; Ei es la migración neta de (i) (emigración - inmigración); BAi es la biomasa acumulada para (i).

La segunda ecuación describe el consumo en cada grupo funcional (i):

$$
\text { Consumo (i) = Producción (i) + Respiración (i) + Alimento no asimilado (i) }
$$

De estas ecuaciones surgen los datos que se requieren para parametrizar el modelo Ecopath. Para cada uno de los grupos funcionales, al menos 3 de los 4 parámetros básicos $(\mathrm{B},(\mathrm{P} / \mathrm{B}),(\mathrm{Q} / \mathrm{B})$ y $\mathrm{EE})$ deben incorporarse en el modelo, además de los valores de la dieta (DC) y capturas por la flota pesquera (Y). Una vez se han incluido los parámetros necesarios, el modelo se equilibra mediante las ecuaciones lineales y se estiman los parámetros básicos desconocidos.

Atendiendo a los supuestos básicos de Ecopath, el modelo se consideró equilibrado cuando los datos de salida del modelo se evaluaron con el análisis de pre-balance (PREBAL), a través de los criterios de Link (2010) para comprobar si el modelo es coherente con los principios y leyes básicas de la ecología de ecosistemas. Ade- 
más, también se utilizó el diagnóstico de las reglas de la ecología y termodinámica que se describen en Darwall et al. (2010) para evaluar que se cumplen una serie de restricciones en los parámetros básicos del modelo.

\subsection{Grupos funcionales}

Para la integración de las especies en los diferentes grupos tróficos, inicialmente se evaluó la disponibilidad de datos de las especies representativas de los fondos rocosos someros del área de estudio, hasta los 40 metros de profundidad. Este enfoque del modelo, excluyó algunos grupos funcionales como aves, tiburones o mamíferos marinos debido a la ausencia de estos niveles tróficos en la metodología de muestreo del área de estudio o que habitan en mar abierto. Los grupos funcionales del modelo se definieron utilizando criterios de similaridad en sus roles ecológicos, sus hábitos alimenticios y su importancia en términos de la pesquería artesanal de El Hierro. Finalmente, el modelo Ecopath contó con 22 grupos funcionales, que incluyeron 2 productores primarios, 2 grupos zooplanctónicos, 7 grupos de invertebrados bentónicos, 10 grupos de peces y un grupo para el detrito (tabla 1). La flota de pesca artesanal también se incluyó en el modelo, a partir de los datos del promedio de descargas del período 2003-2005. Para cada grupo funcional, además de la biomasa, se estimaron los promedios de la producción y consumo (P/B y $\mathrm{Q} / \mathrm{B}$ ), en base a los valores individuales de cada especie ponderadas por su biomasa relativa en el grupo. Por otra parte, se diseńó una matriz de dietas, para representar las relaciones tróficas entre los grupos funcionales del modelo (tabla 2). Todos los parámetros del modelo fueron transformadas a las unidades estandarizadas por Ecopath (toneladas . $\mathrm{km}^{-2}$. año ${ }^{-1}$ ). La composición de especies de todos los grupos funcionales y el origen de los datos para la estimación de los parámetros básicos se detallan en el Anexo A1.

\subsection{CARACTERIZACión trófica DEL ECOSISTEMA}

El modelo Ecopath equilibrado, una vez analizado mediante el diagnóstico PREBAL y las reglas de Darwall et al. 2010, permite caracterizar la estructura trófica del ecosistema en base al nivel trófico de cada grupo, los flujos de energía totales, índices ecológicos, eficiencias y los impactos de la pesca (Christensen y Walters 2004; Christensen et al. 2008; Cury et al. 2005).

\subsubsection{Indices de la Teoría de Ecosistemas}

(1) El índice de pedigrí fue utilizado para describir el origen de los datos y determinar la calidad del modelo (Pauly et al. 2000; Christensen et al. 2008). El rango de este índice varía desde 0 hasta 1 , siendo 1 un indicador de alta calidad del modelo y baja incertidumbre de los datos de entrada. 
(2) El análisis del impacto trófico mixto (ITM) se utilizó para evaluar el impacto trófico en la estructura y funcionamiento del ecosistema. (Ulanowicz y Puccia 1990). Este análisis indica los efectos, positivos o negativos, de un incremento de biomasa de un grupo funcional sobre los demás grupos del ecosistema, incluyendo la flota pesquera.

(3) El índice de especies clave (EC) fue calculado para identificar los grupos funcionales clave del ecosistema, grupos que tiene un rol importante en la red trófica, de acuerdo con la metodología propuesta por Valls et al. (2015).

(4) El nivel trófico (NT) de los grupos funcionales fue calculado para identificar y analizar su posición ecológica en la red trófica (Lindeman 1942). La estructura trófica del ecosistema se representó mediante la agregación de los niveles tróficos en una cadena de flujos (Diagrama de espina) usando el enfoque propuesto por Ulanowicz (1995). Adicionalmente, este diagrama permite cuantificar la eficiencia en la transferencia de energía por cada sección o nivel trófico.

(5) Las transferencias de energía dentro del ecosistema fueron cuantificadas a partir del consumo, la respiración, la importación y exportación y los flujos al detrito. La suma de todos ellos o flujos totales en el ecosistema (FTS) puede ser utilizado como un indicador del tamaño del ecosistema (Christensen y Pauly 1993). En este sentido, un incremento de los FTS indica un incremento de la producción o la tasa de renovación, y el crecimiento del sistema.

Algunos indicadores ecológicos, proporcionados por el modelo, están relacionados con la resiliencia y estado de madurez del sistema de acuerdo con la teoría descrita por Odum (1969). Por ello, los siguientes indicadores fueron incluidos en el análisis del modelo: la relación entre la producción primaria total y la respiración total del sistema (TPP/TR), la cual se aproxima a 1 en ecosistemas maduros mientras que en ecosistemas en desarrollo el valor es superior; la relación entre la producción primaria total y la biomasa total (TPP/TB) con valores bajos en ecosistemas maduros y altos en aquellos en vías de desarrollo y, por último, la producción primaria neta del sistema (PPN), que se aproxima a 0 en ecosistemas maduros.

Adicionalmente, el modelo proporciona otros indicadores ecológicos que nos informan sobre la complejidad, madurez y resiliencia de la red trófica. El índice de omnívoría del sistema (IOS) se relaciona con la especialización trófica y se calcula como la varianza del NT de las presas para cada depredador. El índice de conectancia (IC) muestra la relación entre el número de conexiones existentes entre grupos funcionales y el número de las conexiones posibles (Christensen et al. 2008). La ascendencia (A), que es una medida del grado de desarrollo, organización y eficiencia del ecosistema (Ulanowicz 1986; Ulanowicz y Norden 1990). El límite superior para el tamaño de la A, se conoce como capacidad de desarrollo (C) y, la diferencia entre C y A se denomina superávit $(\mathrm{O})$ (overhead; Ulanowicz 1986), que refleja el potencial de reserva cuando el sistema se enfrenta a perturbaciones externas (Ulanowicz 1986). 


\subsubsection{Indices de reciclaje}

El índice de reciclaje de Finn (IRF) (Finn 1976), representa la fracción de todos los flujos que se reciclan en el sistema. El índice de reciclaje por depredación (IRP) (Christensen et al. 2008) es una modificación del IRF que excluye los grupos que reciclan el detrito. La longitud media de las vías (LMV) se define como el numero promedio de grupos funcionales que un flujo trófico atraviesa dentro del ecosistema (Finn 1976). Valores elevados para IRF, IRP y LMV son indicadores de madurez, resiliencia y estabilidad frente a las perturbaciones del sistema (Christensen 1995), y por ello, también se utilizaron para evaluar el modelo Ecopath.

\subsubsection{Indices del impacto de la actividad pesquera}

Finalmente, se exploraron los indicadores relacionados con el impacto de la actividad pesquera, incluyendo las capturas totales y el nivel trófico promedio de las capturas.

\section{RESULTADOS}

El diagnóstico PREBAL, aplicado bajo las reglas de Link et al. 2010, demostró que los resultados del modelo cumplen con los principios ecológicos. Los parámetros de entrada evaluados (tabla 3 y figura 2) en general, a excepción de los grupos planctónicos, fueron coherentes con los criterios establecidos. Sin embargo, algunos valores de entrada para algunos grupos funcionales resultaron no estar balanceados respecto a los flujos de energía, ya que inicialmente la EE resultó $>1$. Tras balancear el modelo $(\mathrm{EE}<1)$, realizando pequeños ajustes en la matriz de dietas, los resultados del diagnóstico de las reglas de la ecología y termodinámica de Darwall et al. (2010) mostraron consistencia en el modelo con algunas excepciones en los rangos de P/Q para los grupos planctónicos.

Los grupos funcionales del modelo en equilibrio (tabla 4) se integraron en una escala de NTs desde 1 para los productores primarios hasta de 3,89, representado por el grupo de peces piscívoros y macroinvertívoros. La estructura de la red trófica del ecosistema también se representó en un diagrama de flujos (figura 3).

El índice de pedigrí, que califica el modelo en base a la incertidumbre de los datos, obtuvo un valor de 0,69 (tabla 5). Las respuestas más destacadas entre las interacciones tróficas (figura 4), analizadas con el impacto trófico mixto (ITM), se dieron entre Coscinasterias tenuispina frente a Diadema africanum (efecto negativo) y las macroalgas bentónicas frente a Diadema africanum (efecto positivo). Los grupos funcional clave, según el índice de especies clave (EC) definido por Valls et al. (2015), fue el representado por los grupos funcionales piscívoros y macroinvertívoros (figura 5). En cuanto a los flujos de energía y eficiencias entre niveles tróficos representados por el diagrama de espina (Figura 6) se observó que los niveles tróficos (NTs) de nivel 1 (PP y detritus) generan hasta el 72,85\% de los flujos totales 
del sistema (FTS). Los resultados también mostraron que la mayor concentración de biomasa se acumula en los primeros NTs y que la mayor eficiencia energética se da entre el NT II y NT III. Asimismo, las mayores exportaciones y capturas de ecosistema se concentran en el NT II.

Los resultados de los índices ecológicos y los flujos energéticos relacionados con la resiliencia y la madurez del ecosistema (tabla 5) mostraron que de los FTS $\left(5513,21 \mathrm{t} \cdot \mathrm{km}^{-2} \cdot \mathrm{a}^{-1}\right)$ el $35,9 \%$ son flujos al detrito, el $27,7 \%$ por consumo, el $24,9 \%$ se exporta o captura y el $11,5 \%$ corresponden a flujos por respiración. Los índices ecológicos TPP/TR, TPP/TB, y PPN demostraron que el ecosistema presenta valores que lo caracterizan como un sistema inmaduro o en vías de desarrollo. Valores bajos en los índices de reciclaje (IRF, IRP) y en la LMV también indicaron una baja resiliencia del sistema frente a posibles perturbaciones. La especialización trófica del sistema y el número de conexiones entre grupos tróficos, definidos por el IO $(0,19)$ y el IC $(0,23)$ mostraron valores bajos, característicos de un sistema con poca complejidad en su red trófica. El índice que mide el grado de desarrollo, organización y eficiencia del ecosistema (A) obtuvo un valor bajo $(28,76 \%)$ en base a su límite superior de capacidad de desarrollo, y un valor alto $(71,24 \%)$ en el superávit $(\mathrm{O})$.

Por último, en los impactos derivados de la actividad pesquera se cuantificaron un total de $1,486 \mathrm{t} \cdot \mathrm{km}^{-2} \cdot \mathrm{a}^{-1}$, con un NT promedio de las capturas de 2,84.

\section{DISCUSIÓN}

Los resultados de este modelo han demostrado la capacidad de EwE para parametrizar una gran cantidad de información biológica, caracterizando la estructura y funcionamiento del ecosistema modelizado. Los valores de los índices ecológicos estimados por el modelo, han definido el ecosistema de El Hierro desde un enfoque holístico como un sistema de baja complejidad, inmaduro y en vías de desarrollo. Además, la modelización de todos estos parámetros y los resultados obtenidos han identificado las características tróficas más relevantes y las áreas de conocimiento científico que más limitaciones presenta en el ecosistema de la isla de El Hierro.

Este trabajo es el primero que describe en profundidad un modelo trófico en la isla de El Hierro. Con la finalidad de entender los procesos que mantienen al ecosistema, se sintetizó la información biológica y pesquera del área de estudio, confiando en la fiabilidad de los datos para construir un modelo de buena calidad. Sin embargo, la falta de información o presencia de grandes niveles tróficos (mamíferos marinos, aves, tiburones), fue un limitante para construir los grupos funcionales en los niveles superiores del sistema. Dentro de las limitaciones, el pedigrí del modelo presentó unos valores aceptables en cuanto a la calidad de los datos según los índices de pedigrí recopilados de 150 modelos publicados (Morissette 2007). Los resultados del diagnóstico PREBAL resultaron según lo esperado, las relaciones $\mathrm{P} / \mathrm{B}$ y $\mathrm{Q} / \mathrm{B}$ disminuyen conforme aumentan los niveles tróficos, con la excepción de los grupos planctónicos que, además, se representaron en varios subgrupos para destacar la importancia del sistema oligotrófico de las islas Canarias (Hernández, 2016). 
La estructura trófica del ecosistema se pudo determinar a través de niveles tróficos continuos, típica de sistemas en desarrollo, en la que se reflejó una concentración de biomasa y principales flujos en los niveles tróficos bajos e intermedios. Este reparto estructural de biomasa y flujos tróficos es más similar al modelo de la isla de Gran Canaria que al modelo del golfo de Cádiz, donde la concentración de biomasa y flujos principales se reparte también en los niveles tróficos intermedios y superiores.

Las interacciones tróficas más importantes según el análisis de ITM, así como el análisis de EC, señalaron a los grupos funcionales implicados en la aparición de blanquizales en las islas Canarias (Hernández et al. 2008). Estos grupos están representados por el equinodermo Diadema africanum, los depredadores especializados en controlar sus poblaciones (Clemente et al. 2010) y el principal alimento de esta especie de erizo, las macroalgas bentónicas. Por ello, los resultados del modelo nos advierten de la vulnerabilidad del equilibrio que existe entre estos grupos tróficos y del gran impacto que puede suponer la alteración de los mismos.

La caracterización del modelo en base a los índices ecológicos propuestos por Odum (1969), presentó resultados similares respecto a los modelos comparados del Atlántico-oriental. Los flujos totales del sistema (FTS), indicadores del tamańo del sistema, fueron ligeramente inferiores en el modelo de El Hierro respecto a los modelos comparados en el Atlántico-oriental, probablemente por la exclusión de los niveles tróficos más altos (aves, tiburones, mamíferos marinos). Fueron varios los índices ecológicos los que contribuyeron a caracterizar el ecosistema modelizado como en fase de crecimiento o desarrollo, como TPP/TR, TPP/TB, PPN, IO, IC, A y O. Del mismo modo fueron caracterizados los modelos de Gran Canaria y golfo de Cádiz. Sin embargo, aunque el grado de complejidad o desarrollo de los ecosistemas comparados es similar, existen pequeñas diferencias, por ejemplo en los índices de omnivoría (IO) y conectancia (IC) dependiendo de las conexiones tróficas y de los hábitos tróficos de las especies que componen el modelo (especialistas ó generalistas).

Los índices de reciclaje (IRF, IRP) y la LMV presentados por el modelo fueron relativamente bajos, comparados con los obtenidos en los modelos de Gran Canaria y Golfo de Cádiz, lo que se podría ser debido a la presión pesquera que hay en el ecosistema. Las capturas totales de la actividad pesquera artesanal $\left(1,4 \mathrm{t} \cdot \mathrm{km}^{-2} \cdot \mathrm{a}^{-1}\right)$ en el ecosistema sugiere que hay una explotación moderada de los recursos en relación a las capturas totales de la actividad pesquera que opera en el modelo de Gran Canaria o Golfo de Cádiz $\left(4,5 \mathrm{t} \cdot \mathrm{km}^{-2} \cdot \mathrm{a}^{-1}\right)$, donde las flotas pesqueras son de carácter industrial y las capturas son más elevadas. Esta característica también se refleja en el nivel trófico promedio de las capturas, siendo 2,83 en la flota artesanal de La Restinga, frente a 3,43 y 3,32 en Gran Canaria y Golfo de Cádiz, respectivamente. Estos resultados pueden explicarse por la multiespecificidad de las capturas de la flota de El Hierro y en el bajo nivel trófico de la especie que cuenta con más descargas en peso, Sparisoma cretense.

Desde el punto de vista de la modelización trófica, este modelo puede servir de base para realizar exploraciones temporales o crear escenarios simulados, relacionados con los procesos bióticos o abióticos que fuercen cambios en la estructura y 
funcionamiento de la red trófica, como pueden ser diferentes estrategias pesqueras o catástrofes naturales como el vulcanismo reciente. Por lo que creemos que es una herramienta de gran utilidad para el manejo y la conservación de los recursos pesqueros litorales de la isla de El Hierro.

\section{AGRADECIMIENTOS}

A todos los compañeros que de una forma u otra, nos han acompañado durante estos años y han compartido con nosotros campañas, buceos, reuniones, vacilones, etc. A las gentes de La Restinga, un lugar único, maravilloso, donde el tiempo se detiene para dejarnos ver una naturaleza única, indómita, prístina.

\section{CONTRIBUCIÓN DE LOS AUTORES}

Conceptualización: JCM

Metodología y trabajo de campo: JCM y JCH

Análisis de datos: JCM

Preparación del escrito original: JCM

Corrección y edición del escrito definitivo: JCH

RECIBIDO: enero de 2020; ACEPTADO: febrero de 2020 


\section{REFERENCIAS}

Allen, R.R. 1971. Relation between production and biomass. J Fish Res Board Can 28: 1573-1581.

Bortone, S.A., Van Tassell, J., Brito, A., Falcón, J.M. y Bundrick, C.M. 1991. A visual assessment of the inshore fishes and fishery resources off El Hierro, Canary Islands: a baseline survey. Sci Mar 55: 529-541.

Braun. J.G. 1980. Estudios de producción en aguas de las islas Canarias I- Hidrografía, nutrientes y producción primaria. Bol Inst Esp Oceanogr 5: 147-154.

Brito, A., Falcón, J.M., González, G., Pascual, P., Sancho, A., Dorta, C., Hernández, J.C., García, N. 2001e. Valoración de indicadores naturales del efecto reserva en la Reserva Marina de La Restinga-Mar de las Calmas. Departamento de Biología Animal (Ciencias Marinas), Universidad de La Laguna.

Brito, A., Falcón, J.M. y Herrera, R. 2005. Sobre la tropicalización reciente de la ictiofauna litoral de las islas Canarias y su relación con cambios ambientales y actividades antrópicas. Vieraea, 33: 515-525.

Christensen, V. y Pauly, D. 1992. ECOPATH II-a software for balancing steady-state ecosystem models and calculating network characteristics. Ecol Modell 61(3-4): 169-185.

Christensen. V. y Pauly. D. 1993. Trophic models of aquatic ecosystems. (Vol. 26). WorldFish.

Christensen, V. 1995. Ecosystem maturity - towards quantification. Ecol Modell 77: 3-32.

Christensen. V. y Walters, C.J. 2004. Ecopath with Ecosim: methods, capabilities and limitations. Ecol Modell 172: 109-139.

Christensen. V., Walters, C.J., Pauly, D. y Forrest, R. 2008. Ecopath with Ecosim version 6. User guide. Len Oce Futs Proj 235.

Christensen, V. y Walters, C.J. 2011. Progress in the use of ecosystem models for fisheries management. Ecosystem approaches to fisheries: a global perspective. Cambridge University Press, Cambridge 189-205.

Clemente, S., Hernández, J.C., Rodríguez, A. y Brito, A., 2010. Identifying keystone predators and the importance of preserving functional diversity in sublittoral rocky-bottom areas. Marine Ecology Progress Series 413, 55-67.

Couce-Montero, L., Christensen, V. y Castro, J.J. 2015. Effects of small-scale and recreational fisheries on the Gran Canaria ecosystem. Ecol Modell 312:61-76.

Cowan Jr, J.H., Rice, J.C., Walters, C.J., Hilborn, R., Essington, T.E., Day Jr, J.W. y Boswell, K.M. 2012. Challenges for implementing an ecosystem approach to fisheries management. Mar Coast Fish 4(1): 496-510.

Cury, P., Bakun, A., Crawford, R.J.M., Jarre, A., Quinones, R.A., Shannon, L.J. y Verh-eye, H.M. 2000. Small pelagics in upwelling systems: patterns of interaction and structural changes in "wasp-waist» ecosystems. ICES J Mar Sci 57: 603-618.

Cury, P.M., Mullon, C., Garcia, S.M. y Shannon, L.J. 2005. Viability theory for an ecosystem approach to fisheries. ICES J Mar Sci J 62: 577-584.

Darwall, W.R., Allison, E.H., Turner, G.F. y Irvine, K. 2010. Lake of flies, or lake of fish? A trophic model of Lake Malawi. Ecol Modell 221(4): 713-727. 
De La Cruz-Modino, R. 2011. Turismo, pesca y gestión de recursos. Aportaciones desde La Restinga y L'Estartit. Premio de Investigación Cultural Marqués de Lozoya 2009. Secretaría General Técnica. Centro de Publicaciones. Ministerio de Educación, Cultura y Deporte. 225 pp.

De León, A.R. y Braun, J.G. 1973. Ciclo anual de la producción primaria y su relación con los nutrientes en aguas canarias. Bol Inst Esp Oceanogr 167, 1-24.

Falcón, J.M., Bortone, S.A, Brito, A. Bundrick, C.M. 1996. Structure of and relationships within and between the littoral, rock-substrate fish communities off four islands in the Canarian Archipelago. Mar. Biol. 125(2), 215-231.

Falcón, J.M. 2016. Ictiofauna de las islas canarias. Análisis biogeográfico. 2016. Doctoral thesis. Universidad de La Laguna. Tenerife, islas Canarias, 310 pp.

FInN, J.T. 1976. Measures of ecosystem structure and function derived from analysis of flows. J Theor Biol 56:363-380.

Guénette, S. y Morato, T., 2001. The Azores Archipelago, 1997. Fish Cent Res Rep, 9(4), 241-270.

Hernández, C.A. 2016. Sistema de carbono y productividad primaria en zonas costeras de Canarias en un contexto de Cambio Global. Doctoral thesis. Universidad de La Laguna, Tenerife, islas Canarias, 210 pp.

Heymans, J.J., Coll, M., Link, J.S., Mackinson, S., Steenbeek, J., Walters, C. y ChristenSEN, V. 2016. Best practice in Ecopath with Ecosim food-web models for ecosystem-based management. Ecol Modell 331: 173-184.

Lindeman, R.L. 1942. The trophic-dynamic aspect of ecology. Ecol 23: 399-417.

LINK, J.S. 2010. Adding rigor to ecological network models by evaluating a set of pre-balance diagnostics: a plea for PREBAL. Ecol Modell 221: 1580-1591.

Odum, E.P. 1969. The strategy of ecosystem development. Science 164: 262-270.

Pauly, D., Christensen, V. y Walters, C. 2000. Ecopath, Ecosim, and Ecospace as tools for evaluating ecosystem impact of fisheries. ICES J Mar Sci: J Conseil 57: 697-706.

Polovina, J.J. 1984. Model of a coral reef ecosystem. Coral reefs 3: 1-11.

SAngil, C., SAnson, M. y Afonso, J. 2011. La vegetación sublitoral de sustratos rocosos en El Hierro (islas Canarias): relación entre variables ambientales y estructura de las comunidades de macroalgas. Vieraea, 39, 167-182.

Stanford, R., Lunn, K. y Guénette, S., 2001. A preliminary ecosystem model for the Atlantic coast of Morocco in the mid-1980 s. Fish Cent Res Rep, 9 (4), 314-344.

Stobberup, K.A., Ramos, V.D.M. y Coelho, M.L. 2004. Ecopath model of the Cape Verde coastal ecosystem. In West Afr Mar Ecos, MLD Palomares and D. Pauly.

Torres, M.A., Coll, M., Heymans, J.J., Christensen, V. y Sobrino, I. 2013. Food-web structure of and fishing impacts on the Gulf of Cadiz ecosystem (South-western Spain). Ecol Modell 265: 26-44.

Tuya, F., Boyra, A., Sanchez-Jerez, P., Barbera, C., Haroun, R.J. 2004. Relationships between rockyreef fish assemblages, the sea urchin Diadema antillarum and macroalgae throughout the Canarian Archipelago. Mar Ecol Prog Ser, 278, 157-169.

Ulanowicz, R.E. 1986. Growth and Development: Ecosystems Phenomenology. Springer 203 pp.

UlANOwICZ, R.E. 1995. Utricularia's secret: the advantage of positive feedback in oligotrophic environments. Ecol Modell 79 (1-3): 49-57. 
Ulanowicz, R.E. y Puccia, C.J. 1990. Mixed trophic impacts in ecosystems. Coenoses 5: 7-16.

Ulanowicz, R.E. y Norden, J.S. 1990. Symmetrical overhead in flow networks. Int J Syst Sci 21: 429-437.

Valls, A., Coll, M. y Christensen, V. 2015. Keystone species: toward an operational concept for marine biodiversity conservation. Ecol Monogr, 85(1), 29-47.

\section{TABLAS}

\begin{tabular}{|c|c|c|c|c|c|c|}
\hline & \multicolumn{6}{|c|}{ 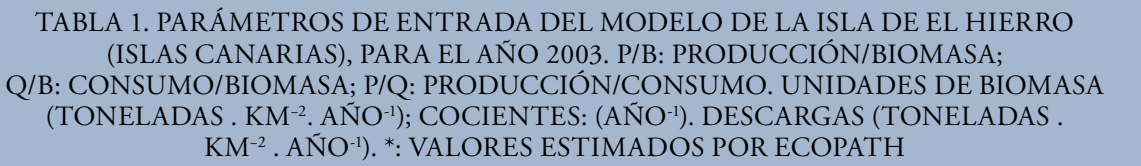 } \\
\hline & Grupo funcional & BIOMASA & $\mathrm{P} / \mathrm{B}$ & $\mathrm{Q} / \mathrm{B}$ & $\mathrm{P} / \mathrm{Q}$ & Descargas \\
\hline 1 & Fitoplancton & 7,40 & 187,78 & & & \\
\hline 2 & Macroalgas bentónicas & 72,26 & 10,16 & & & \\
\hline 3 & Bacterioplancton & 9,60 & 27,93 & $69,82^{*}$ & 0.40 & \\
\hline 4 & Mesozooplancton & 1,280 & $38,10^{*}$ & 95,25 & 0.40 & \\
\hline 5 & Meiofauna bentónica & 15,35 & 6,55 & 18,15 & & \\
\hline 6 & Macroinvertebrados detritívoros & 33,46 & 0,945 & 7,49 & & \\
\hline 7 & Diadema africanum & 2,060 & 1,38 & 8,15 & & \\
\hline 8 & Coscinasterias tenuispina & 1,250 & 1,24 & 6,05 & & \\
\hline 9 & Macrocrustáceos & 7,65 & 1,18 & 11,59 & & \\
\hline 10 & Moluscos & 6,35 & 1,97 & 8,56 & & \\
\hline 11 & Cefalópodos & 0,85 & 1,47 & 5,25 & & \\
\hline 12 & Sarpa salpa & 0,10 & 2,40 & 19,80 & & 0,10 \\
\hline 13 & Espárdios & 0,59 & 0,57 & 5,51 & & \\
\hline 14 & Sparisoma cretense & 0,29 & 5,06 & 13,50 & & 0,77 \\
\hline 15 & Peces planctívoros & 0,95 & 0,68 & 6,89 & & \\
\hline 16 & Peces microinvertívoros & 0,37 & 0,75 & 7,49 & & \\
\hline 17 & Peces piscívoros y microinvertívoros & 1,47 & 0,94 & 5,87 & & 0,12 \\
\hline 18 & Peces macroinvertívoros & 0,14 & 1,35 & 4,87 & & 0,05 \\
\hline 19 & Pequeños piscívoros y macroinvertívoros & 0,39 & 0,59 & 4,53 & & 0,13 \\
\hline 20 & Piscívoros y macroinvertívoros & 0,27 & 1,01 & 2,97 & & 0,22 \\
\hline 21 & Morenas & 0,07 & 1,43 & 3,74 & & 0,07 \\
\hline 22 & Detritus & 21,18 & & & & \\
\hline
\end{tabular}




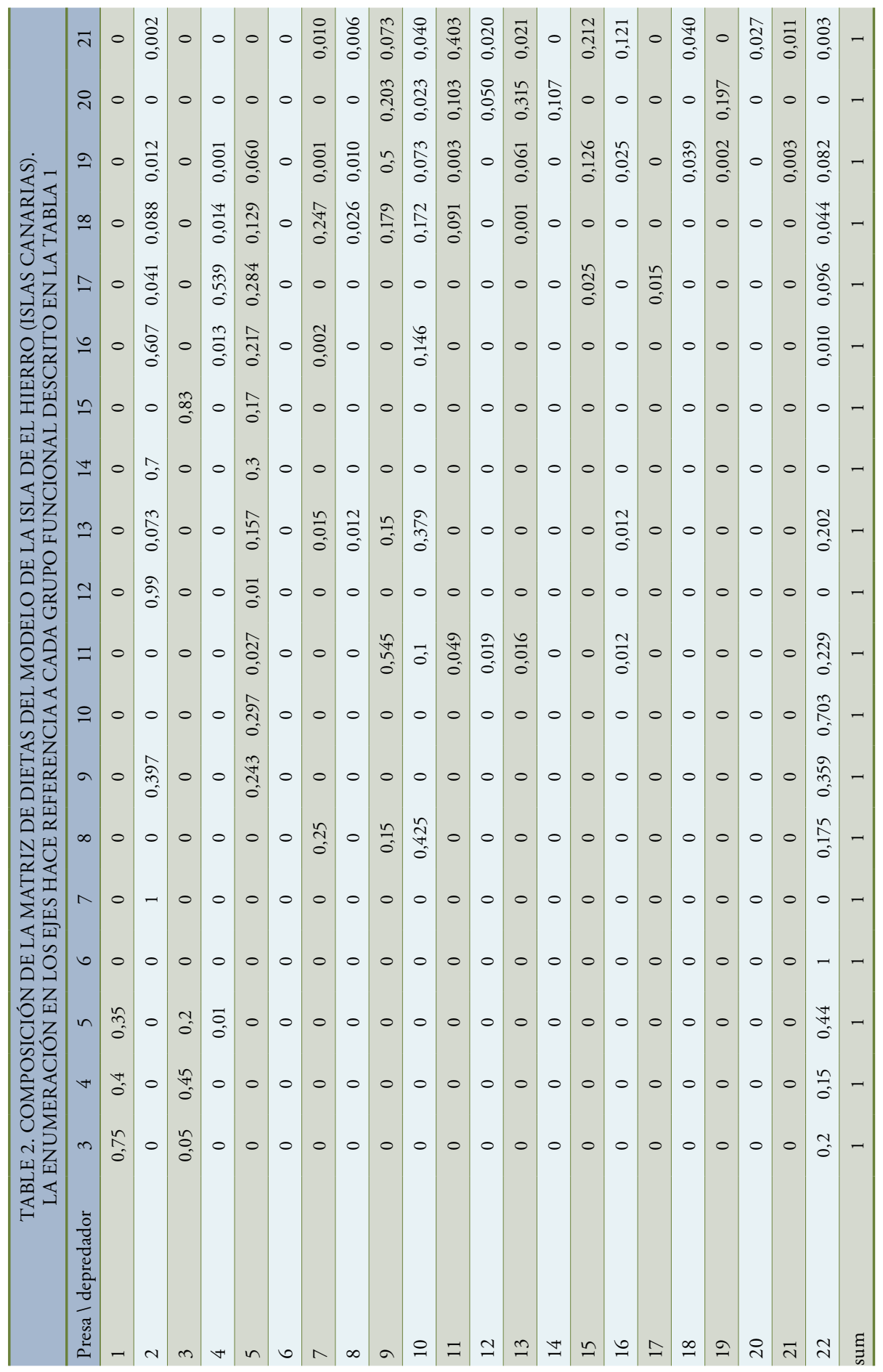


TABLA 3. DIAGNÓSTICOS UTILIZADOS PARA BALANCEAR EL MODELO DE LA ISLA

DE EL HIERRO (ISLAS CANARIAS) EN BASE A LAS REGLAS DE LINK (2010) Y DARWALL

ET AL. 2010. LA LISTA DE VERIFICACIÓN PRESENTA LAS REGLAS Y EL ESTADO DE

LAS MISMAS EN EL MODELO.* LAS POSIBLES OBSERVACIONES PARA CADA UNA DE

LAS REGLAS APARECEN EN LOS COMENTARIOS. B: BIOMASA, Q: CONSUMO,

P: PRODUCCIÓN, PP: PRODUCCIÓN PRIMARIA, R: RESPIRACIÓN, NT: NIVEL TRÓFICO, EE: EFICIENCIA ECOTRÓFICA

\begin{tabular}{lcc}
$\begin{array}{c}\text { Clase de } \\
\text { Diagnóstico }\end{array}$ & Reglas & Ostado \\
\hline 1- PREBal & & \\
\hline
\end{tabular}

\begin{tabular}{|c|c|c|c|}
\hline \multirow{3}{*}{$\begin{array}{l}\text { B a través de gru- } \\
\text { pos y NTs }\end{array}$} & $\begin{array}{l}\text { Abarca un rango de 5-7 órdenes de mag- } \\
\text { nitud }\end{array}$ & $\mathrm{OK}$ & \\
\hline & $\begin{array}{l}\mathrm{L} \text { pendiente disminuye un } 5-10 \% \text { a lo largo } \\
\text { que incrementan los NTs }\end{array}$ & $\mathrm{OK}$ & \\
\hline & $\begin{array}{l}\text { Pocos grupos por encima o por debajo de la } \\
\text { pendiente de la línea }\end{array}$ & OK & $\begin{array}{l}\text { Excepto Sarpa salpa y Sparisoma } \\
\text { cretense }\end{array}$ \\
\hline \multirow[b]{2}{*}{ Relación de B } & B del depredador menor que la de su presa & $\mathrm{OK}$ & $\begin{array}{l}\text { Excepto grupos planctónicos } \\
\text { (sistema oligotrófico) }\end{array}$ \\
\hline & $\begin{array}{l}\text { Distribución equitativa de B en NTs } \\
\text { comparables en la principales vías de flujo } \\
\text { trófico }\end{array}$ & $\mathrm{OK}$ & \\
\hline \multirow{2}{*}{$\begin{array}{l}\text { Tasas vitales a } \\
\text { través de grupos } \\
\text { y NTs }\end{array}$} & $\mathrm{Q} / \mathrm{B}$ y $\mathrm{P} / \mathrm{B}$ conforme aumenta NTs & OK & \\
\hline & $\begin{array}{l}\text { Pocos grupos notablemente por encima o } \\
\text { por debajo de la pendiente de la línea }\end{array}$ & $\mathrm{OK}$ & $\begin{array}{l}\text { Excepto macroinvertebrados de- } \\
\text { tritívoros y Diadema africanum }\end{array}$ \\
\hline \multirow{4}{*}{ Tasas vitales } & $\begin{array}{l}\text { Relación de tasas vitales de depredador } \\
\text { menor que la de sus presas }\end{array}$ & OK & \\
\hline & $\mathrm{P} / \mathrm{B}$ a través de grupos menor que la PP & $\mathrm{OK}$ & \\
\hline & P/Q menor a 1 para cada grupo & $\mathrm{OK}$ & \\
\hline & P/R menor a 1 para cada grupo & OK & $\begin{array}{l}\text { Excepto bacterio y mesozoo- } \\
\text { plancton }\end{array}$ \\
\hline \multicolumn{4}{|c|}{ 2- Reglas ecológicas y termodinámicas } \\
\hline \multirow{6}{*}{$\begin{array}{l}\text { A partir de } \\
\text { Darwall et al., } \\
\text { 2010: }\end{array}$} & $\begin{array}{l}\text { EE estimada }<1 \text { para todos los grupos } \\
\text { funcionales }\end{array}$ & OK & \\
\hline & $\begin{array}{l}\text { Valores de P/Q (relación producción/con- } \\
\text { sumo o eficiencia bruta de conversión de } \\
\text { alimento (GE) está entre } 0,1 \text { y } 0,3 \text { con la } \\
\text { excepción de algunos grupos de crecimien- } \\
\text { to rápido) }\end{array}$ & $\mathrm{OK}$ & $\begin{array}{l}\text { Excepto grupos planctónicos } \\
\text { (sistema oligotrófico) }\end{array}$ \\
\hline & $\mathrm{R} / \mathrm{A}$ (respiración/asimilación alimento) $<1$ & $\mathrm{OK}$ & \\
\hline & $\begin{array}{l}\mathrm{R} / \mathrm{B} \text { (respiración/biomasa) está entre } 1 \text { y } 10 \\
\text { para peces y valores mayores para pequeños } \\
\text { organismos }\end{array}$ & $\mathrm{OK}$ & Excepto Sarpa salpa \\
\hline & $\begin{array}{l}\text { EN (eficiencia neta conversión de alimento) } \\
<\text { GE }\end{array}$ & $\mathrm{OK}$ & \\
\hline & $\mathrm{P} / \mathrm{R}$ (producción/respiración) < 1 & OK & \\
\hline
\end{tabular}




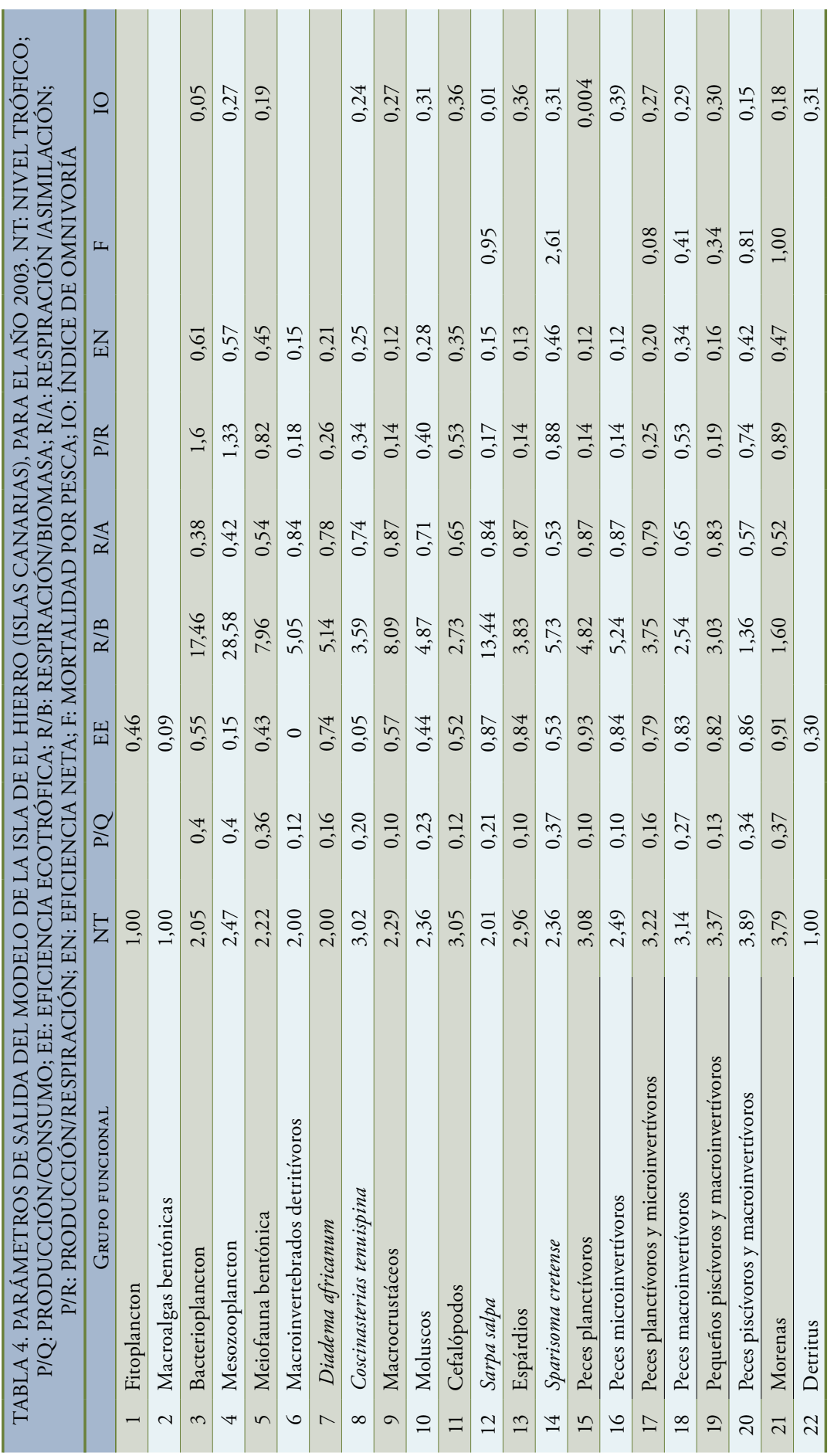




\begin{tabular}{|c|c|c|}
\hline \multicolumn{3}{|c|}{$\begin{array}{l}\text { TABLE 5. RESUMEN DE LOS FLUJOS BÁSICOS E ÍNDICES ECOLÓGICOS DEL MODELO } \\
\text { DE LA ISLA DE EL HIERRO (ISLAS CANARIAS) }\end{array}$} \\
\hline INDICADORES ECOLÓGICOS & VALORES & UNIDADES \\
\hline Ecopath índice pedigrí & 0,690 & \\
\hline Flujos totales (FTS) & 5513,1 & $\mathrm{t} \cdot \mathrm{km}^{-2} \cdot \mathrm{a}^{-1}$ \\
\hline Sum de todos los consumos & 1524,48 & $\mathrm{t} \cdot \mathrm{km}^{-2} \cdot \mathrm{a}^{-1}$ \\
\hline Sum de todas las exportaciones & 1376,38 & $\mathrm{t} \cdot \mathrm{km}^{-2} \cdot \mathrm{a}^{-1}$ \\
\hline Sum de todos los flujos respiración & 637,52 & $\mathrm{t} \cdot \mathrm{km}^{-2} \cdot \mathrm{a}^{-1}$ \\
\hline Sum de todos los flujos al detrito & 1974,82 & $\mathrm{t} \cdot \mathrm{km}^{-2} \cdot \mathrm{a}^{-1}$ \\
\hline Producción primaria total/Respirción total (TPP/TR) & 3,15 & $\mathrm{t} \cdot \mathrm{km}^{-2} \cdot \mathrm{a}^{-1}$ \\
\hline Producción primaria neta (PPN) & 2013,65 & $\mathrm{t} \cdot \mathrm{km}^{-2} \cdot \mathrm{a}^{-1}$ \\
\hline Producción primaria total /Biomasa total (TPP/TB) & 7,88 & $\mathrm{t} \cdot \mathrm{km}^{-2} \cdot \mathrm{a}^{-1}$ \\
\hline Biomasa total/Flujos totales (TB/FTS) & 0,027 & $y^{-1}$ \\
\hline Índice de reciclaje de Finn (IRF) & 6,26 & $\%$ \\
\hline Índice de reciclaje por depredación (IRP) & 2,16 & $\%$ \\
\hline Longitud media de las vías (LMV) & 2,73 & \\
\hline Ascendencia (A) & 28,76 & $\%$ \\
\hline Capacidad de desarrollo (C) & 21982 & flowbits \\
\hline Superávit $(\mathrm{O})$ & 71,24 & $\%$ \\
\hline Índice de omnivoría del sistema (IOS) & 0,191 & \\
\hline Índice de conectancia (IC) & 0,230 & \\
\hline Eficiencia total de todos los flujos (TTE) & 10,10 & $\%$ \\
\hline Capturas totales & 1,486 & $\mathrm{t} \cdot \mathrm{km}^{-2} \cdot \mathrm{a}^{-1}$ \\
\hline Nivel trófico promedio de las capturas & 2,839 & \\
\hline
\end{tabular}




\section{FIGURAS}

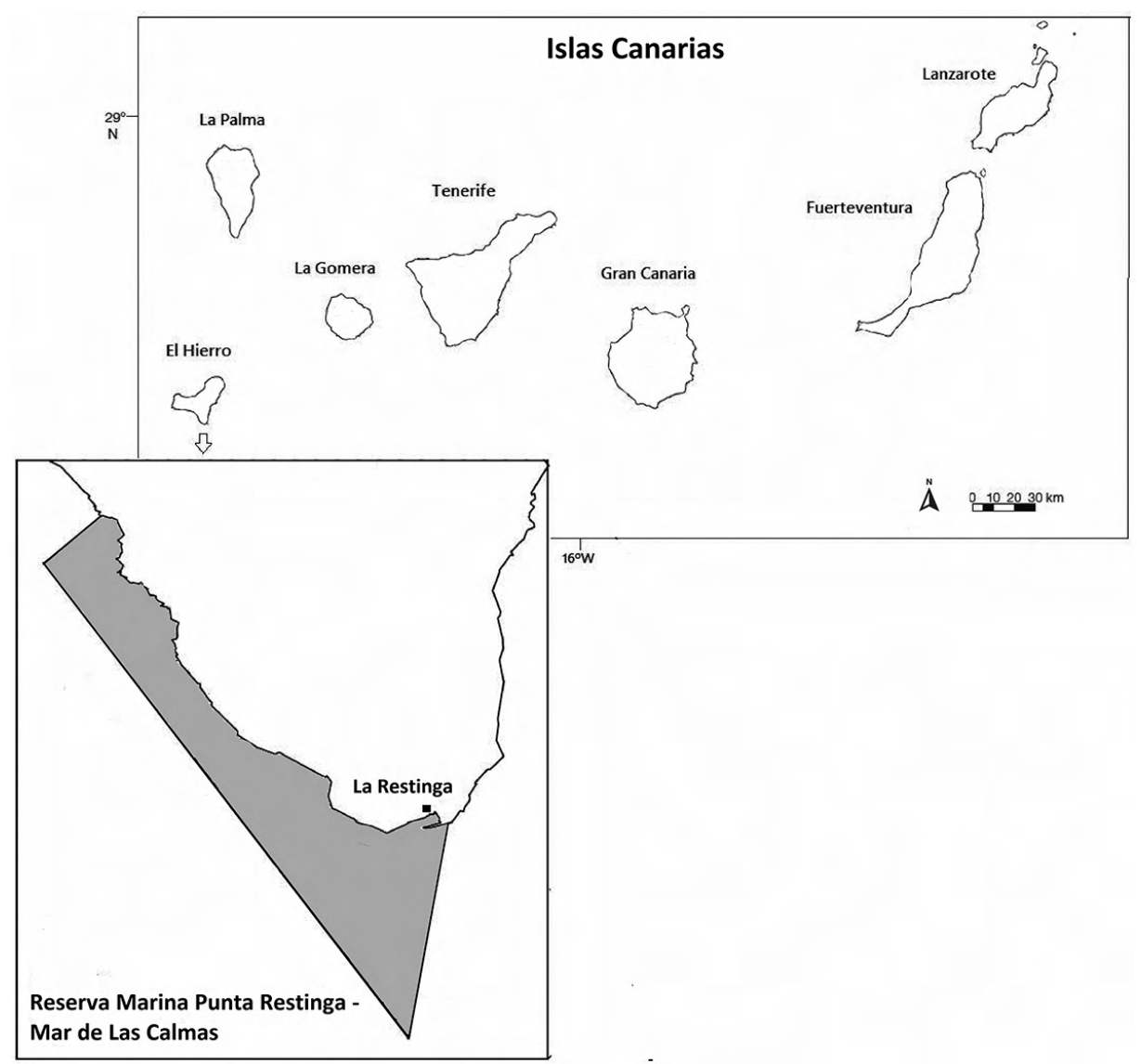

Figura 1. Mapa de las Islas Canarias, mostrando la localización de la Reserva de Punta Restinga -Mar de Las Calmas-. En gris, el área modelizada con Ecopath. 


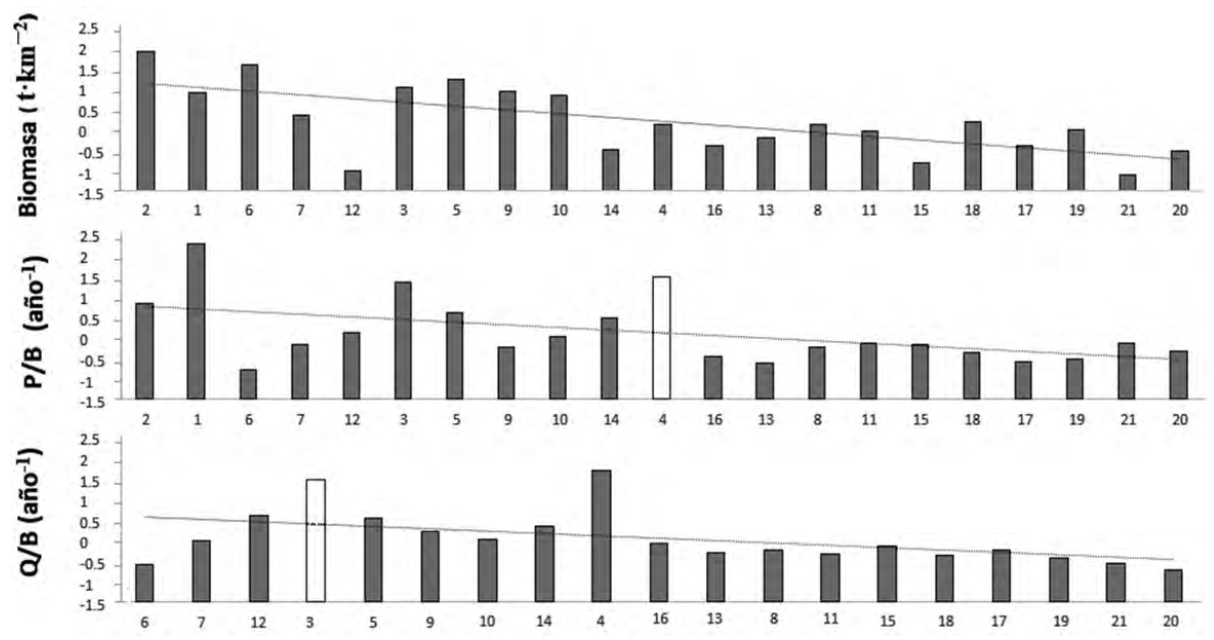

Figura 2. Relaciones vitales (en escala logarítmica) a través de los grupos y niveles tróficos del modelo de la isla de El Hierro (Islas Canarias), para el año 2003. Los niveles tróficos (NTs) aumentan de izquierda a derecha y se presentan las líneas de tendencia. Los números hacen referencia a cada grupo funcional descrito en la tabla 1. B: biomasa, Q: consumo, P: producción

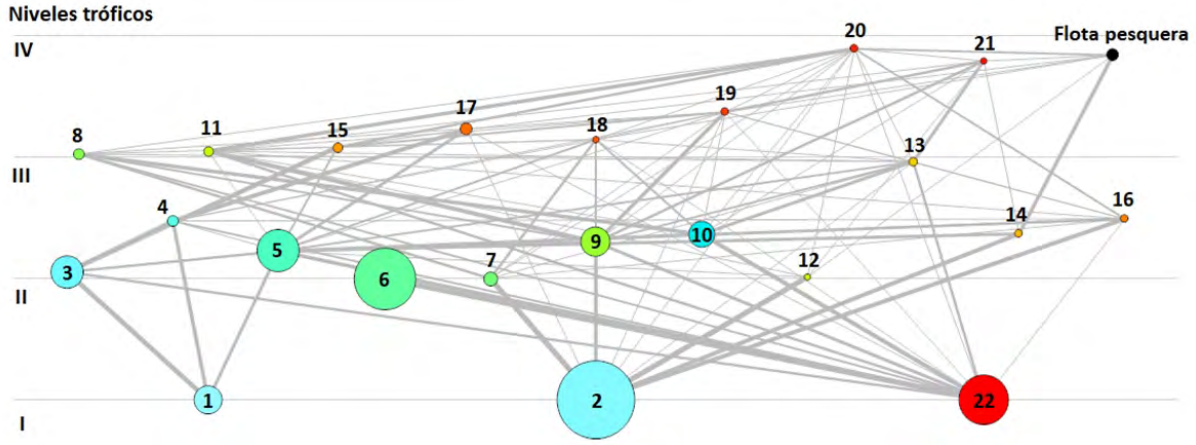

Figura 3. Diagrama de flujo y niveles tróficos de los 22 grupos funcionales incluidos en el modelo de la isla de El Hierro (Islas Canarias), para el año 2003. Los círculos son proporcionales a la biomasa de cada grupo funcional y las líneas representan las conexiones tróficas entre grupos.

El grosor de las líneas de conexión es proporcional a la magnitud de los flujos tróficos.

Los números hacen referencia a cada grupo funcional descrito en la tabla 1. 


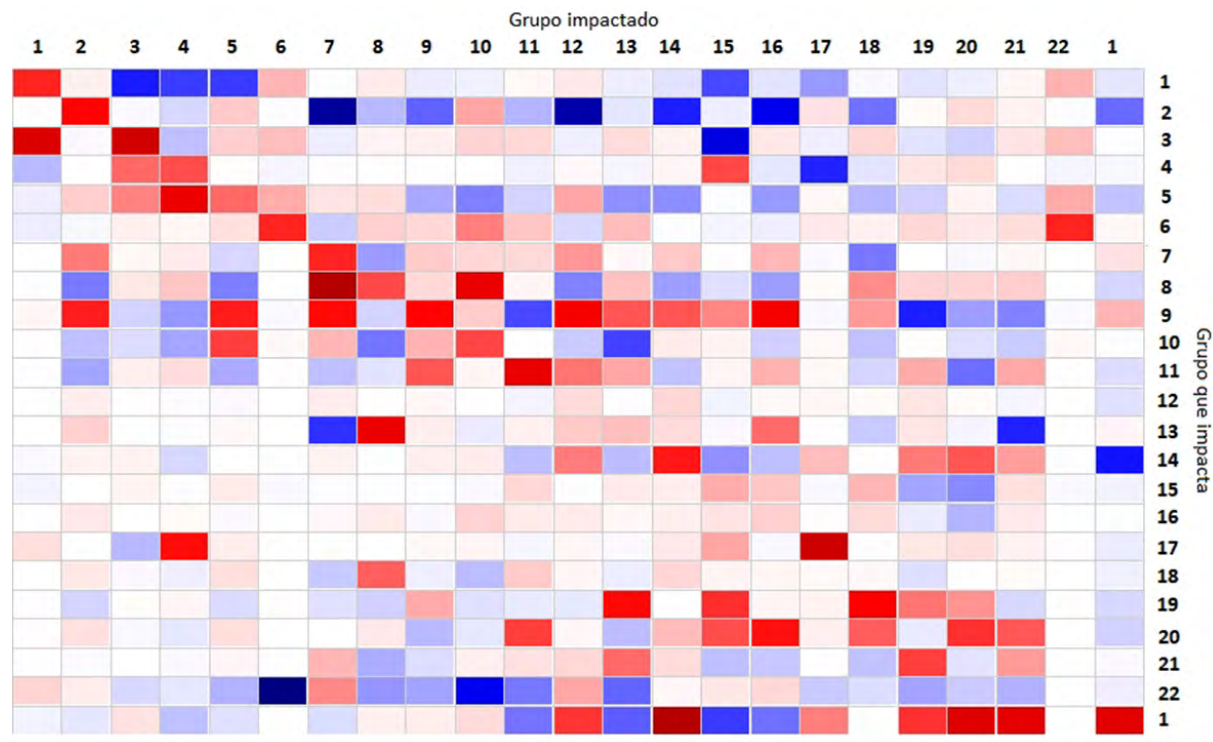

Figura 4. Impactos tróficos mixtos entre los grupos funcionales del modelo de la isla de El Hierro

(Islas Canarias), para el año 2003. Las tonalidades azules indican un impacto relativo neto positivo y las tonalidades rojas un impacto relativo neto negativo. Los números hacen referencia a cada grupo funcional descrito en la tabla 1.

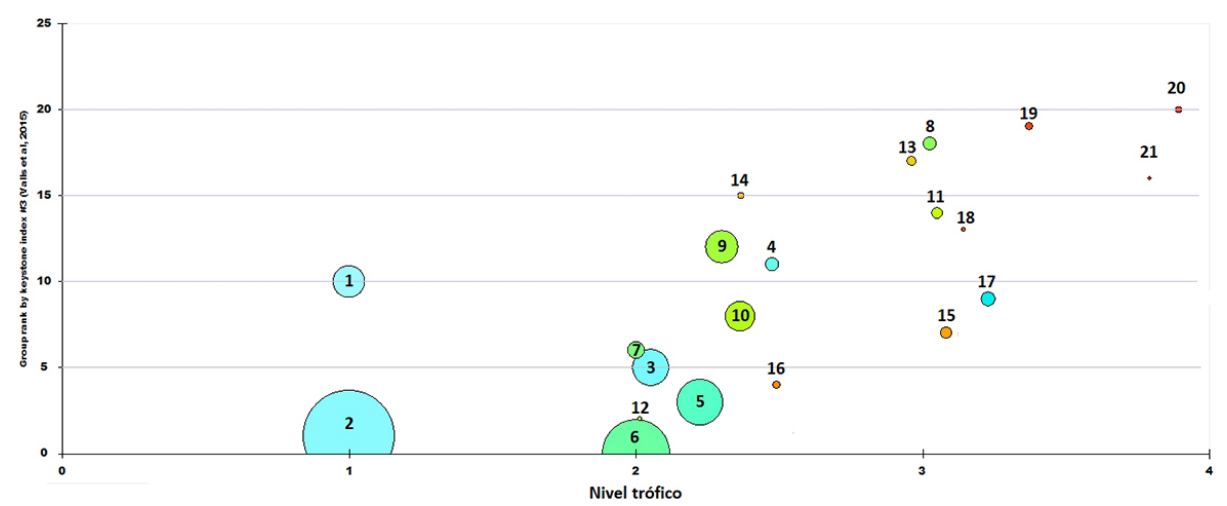

Figura 5. Índice de especies clave (EC) sensu Valls et al. 2015 para los grupos funcionales incluidos en el modelo de la isla de El Hierro (Islas Canarias), para el año 2003. El tamaño de los círculos es proporcional a la biomasa de cada grupo funcional. Los números hacen referencia a cada grupo funcional descrito en la tabla 1. 

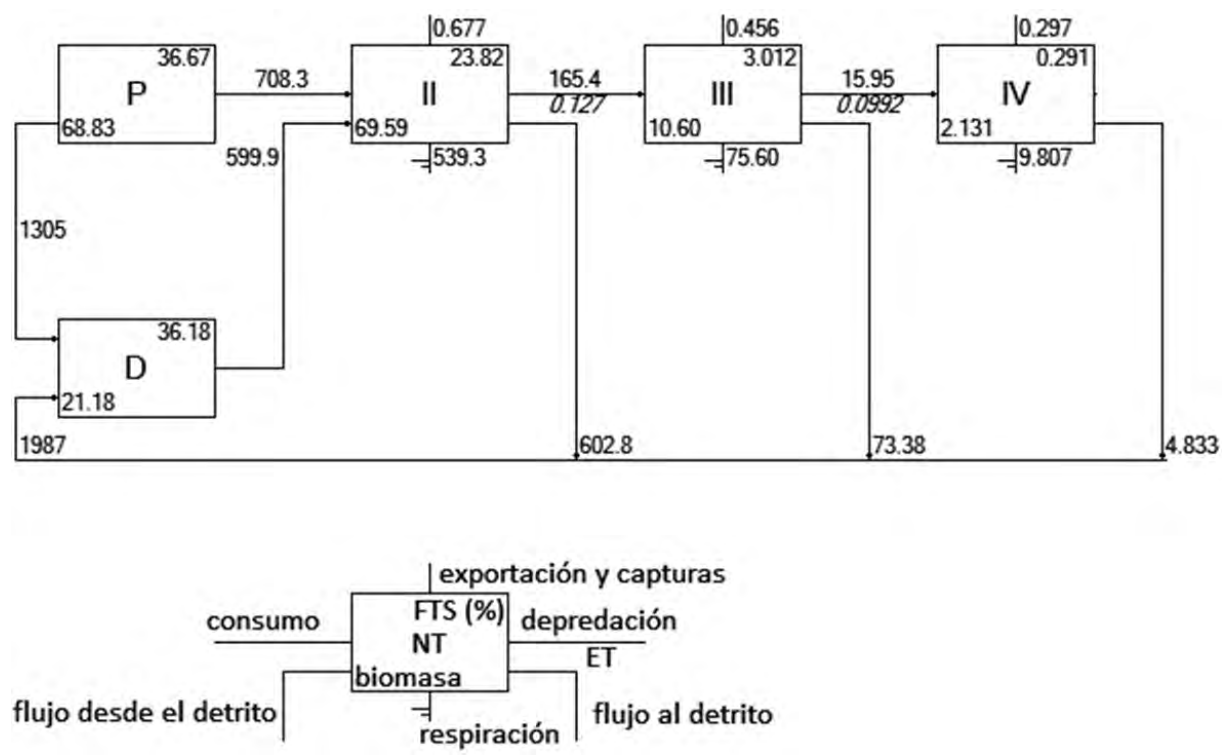

Figura 6. Diagrama de espina de los flujos y eficiencias entre los niveles tróficos del modelo de la isla de El Hierro (Islas Canarias), para el año 2003. Las unidades de los flujos están en toneladas . $\mathrm{km}^{-2}$. año ${ }^{-1} \mathrm{P}$ : productores, $\mathrm{D}$ : detrito, NT: nivel trófico. 


\section{APÉNDICE A}

\begin{tabular}{|c|c|c|}
\hline \multicolumn{3}{|c|}{$\begin{array}{l}\text { TABLA A1. DESCRIPCIÓN DE LOS PARÁMETROS DE ENTRADA Y LAS REFERENCIAS } \\
\text { POR GRUPOS FUNCIONALES DEL MODELO DE LA ISLA DE EL HIERRO. } \\
\text { LOS PORCENTAJES REPRESENTAN LA COMPOSICIÓN DE ESPECIES } \\
\text { EN TÉRMINOS DE PESO PARA CADA GRUPO FUNCIONAL }\end{array}$} \\
\hline Grupo funcional & Parámetro & REFERENCIAS Y ORIGEN DE LOS DATOS \\
\hline 1. Fitoplancton & $\mathrm{B}$ & $\begin{array}{l}\text { - Arístegui et al. (2001) } \\
\text { Factor de conversión utilizado para transformar } \\
\text { unidades de carbono a unidades de materia orgáni- } \\
\text { ca (Dalsgaard y Pauly 1997) } \\
\text { - Arístegui et al. (2001) }\end{array}$ \\
\hline
\end{tabular}

\section{Macroalgas bentónicas}

$100 \%$ Lobophora variegata
Hábitat del área en el modelo: $84.5 \%$

B - Muestreos mediante buceo autónomo (2003) (método de transecto $50 \times 50 \mathrm{~cm}-0,25 \mathrm{~cm} 2$ ) en localidades del área de estudio para obtener porcentajes de cobertura y el peso húmedo por área (Tuya y Haroun 2006). 10 réplicas que abarcan el rango batimétrico del modelo $(0-40 \mathrm{~m})$ en cada localidad de estudio.

- Hernández (2016)

\section{Bacterioplancton}

B

- Estimada a partir de la relación BOC/POC (Bacterial organic carbon / Phytoplankton organic carbon) en las Islas Canarias (Arístegui et al. 2001).

Factor de conversión utilizado para transformar unidades de carbono a unidades de materia orgánica (Sherr y Sherr 1984).

$\mathrm{P} / \mathrm{B} \quad-$ Bode et al. (2001)

$\mathrm{Q} / \mathrm{B} \quad$ - Estimado a partir de $\mathrm{P} / \mathrm{Q}=0,4$

U/Q - Asunción (Christensen et al. 2008)

Dieta - Arístegui et al. (2001)

$\begin{array}{ll}\text { B } & - \text { Arístegui } \text { et al. (2001) } \\ \text { P/B } & \text { - Huggett } \text { et al. }(2009) \\ \text { Q/B } & \text { - Hernández-León } \text { et al. (2001) } \\ \text { U/Q } & \text { - Asunción (Christensen et al. 2008) } \\ \text { Dieta } & \text { - Arístegui } \text { et al. (2001) }\end{array}$

B

$\mathrm{P} / \mathrm{B}$

$\mathrm{Q} / \mathrm{B}$

$\mathrm{U} / \mathrm{Q}$

Dieta
- Muestreos mediante buceo autónomo en el área de estudio

- Brey (2001)

- Estimado a partir de Moens y Vincx (1996)

- Asunción (Christensen et al. 2008)

- Estimado a partir de Moens y Vincx (1996)

\section{Macroinvertebrados detritívoros}

$98,95 \%$

Holoturia sanctori

$1,05 \%$
Hermodice carunculata

- Estimada a partir de las densidades de Ortega et al. (2009) en la Isla de El Hierro; Navarro et al. (2013) (peso promedio de la especie en la isla de Gran Canaria); Conand (1993) (relación talla-peso para Holoturia atra)

- Brey (2001)

$\begin{array}{ll}\text { P/B } & - \text { Brey (2001) } \\ \text { Q/B } & - \text { Pauly et al. (1993); Opitz (1996) } \\ \text { U/Q } & - \text { Asunción (Christensen et al. 2008) } \\ \text { Dieta } & - \text { Asunción: se alimenta exclusivamente del detrito }\end{array}$




\begin{tabular}{|c|c|c|}
\hline 7. Diadema africanum & $\begin{array}{l}\mathrm{P} / \mathrm{B} \\
\mathrm{Q} / \mathrm{B} \\
\mathrm{U} / \mathrm{Q} \\
\text { Dieta }\end{array}$ & $\begin{array}{l}\text { - Muestreos mediante buceo autónomo (transecto } \\
\text { lineal) en el área de estudio (densidades) y relación } \\
\text { talla-peso de la especie (Hernández et al. 2005). } \\
10 \text { réplicas que abarcan el rango batimétrico del } \\
\text { modelo (0-40m) en cada localidad de estudio. } \\
\text { - Ebert (1985) } \\
\text { - Tuya et al. (2001) } \\
\text { - Asunción (Christensen et al. 2008) } \\
\text { - Asunción: Se alimenta exclusivamente de macro- } \\
\text { algas }\end{array}$ \\
\hline 8. Coscinasterias tenuispina & $\begin{array}{l}\mathrm{P} / \mathrm{B} \\
\mathrm{Q} / \mathrm{B} \\
\mathrm{U} / \mathrm{Q} \\
\text { Dieta }\end{array}$ & $\begin{array}{l}\text { - Muestreos mediante buceo autónomo (transecto } \\
\text { lineal) en el área de estudio (densidades) y relación } \\
\text { talla-peso muestreada en un área similar (isla de } \\
\text { Tenerife- Islas Canarias). } \\
\text { - Brey (2001) } \\
\text { - Brey (2001) } \\
\text { - Asunción (Christensen et al. 2008) } \\
\text { - Estimado a partir de Ortega et al. (2011) }\end{array}$ \\
\hline $\begin{array}{l}\text { 9. Macrocrustáceos } \\
\begin{array}{ll}22,38 \% & \text { Percnon gibbesi } \\
77,62 \% & \text { Stenorhynchus lanceolatus }\end{array}\end{array}$ & $\begin{array}{l}\mathrm{P} / \mathrm{B} \\
\mathrm{Q} / \mathrm{B} \\
\mathrm{U} / \mathrm{Q} \\
\text { Dieta }\end{array}$ & $\begin{array}{l}\text { - Estimada a partir de las densidades de Ortega et } \\
\text { al. (2009) en la Isla de El Hierro; Robinson et al. } \\
\text { (2010) (relación talla-peso); Sciberras et al. (2008) } \\
\text { (peso corporal promedio por especie). } \\
\text { - Brey (2001) } \\
\text { - Pauly et al. (1993); Opitz (1996) } \\
\text { - Asunción (Christensen } \text { et al. 2008) } \\
\text { - Sciberras et al. (2008) }\end{array}$ \\
\hline $\begin{array}{l}\text { 10. Moluscos } \\
\text { 83,16\% Pagurus prideaux } \\
\text { 11,25\% Stramonita haemastoma } \\
\text { 5,59\% Dardanus sp. }\end{array}$ & $\begin{array}{l}\mathrm{B} \\
\mathrm{P} / \mathrm{B} \\
\mathrm{Q} / \mathrm{B} \\
\mathrm{U} / \mathrm{Q} \\
\text { Dieta }\end{array}$ & $\begin{array}{l}\text { - Estimada a partir de las densidades de Ortega et al. } \\
\text { (2009) en la Isla de El Hierro } \\
\text { - De otro modelo (Couce-Montero et al. 2015) } \\
\text { - De otro modelo (Couce-Montero et al. 2015) } \\
\text { - Asunción (Christensen } \text { et al. 2008) } \\
\text { - Ramsay et al. (1996) }\end{array}$ \\
\hline $\begin{array}{l}\text { 11. Cefalópodos } \\
50 \% \text { Octopus vulgaris } \\
50 \% \text { Sepia officinalis }\end{array}$ & $\begin{array}{l}\mathrm{P} / \mathrm{B} \\
\mathrm{Q} / \mathrm{B} \\
\mathrm{U} / \mathrm{Q} \\
\text { Dieta }\end{array}$ & $\begin{array}{l}\text { - Estimada a partir de las densidades de Ortega } \\
\text { et al. (2009) en la Isla de El Hierro; López (2000) } \\
\text { (relación talla-peso y peso medio corporal en la isla } \\
\text { de Gran Canaria) } \\
\text { - De otro modelo (Couce-Montero et al. } 2015) \\
\text { - De otro modelo (Couce-Montero et al. 2015) } \\
\text { - Asunción (Christensen } \text { et al. 2008) } \\
\text { - Castro y Guerra (1990); López (2000) }\end{array}$ \\
\hline 12. Sarpa salpa & $\begin{array}{l}\mathrm{Q} / \mathrm{B} \\
\mathrm{U} / \mathrm{Q} \\
\text { Dieta } \\
\text { Descargas }\end{array}$ & $\begin{array}{l}\text { - Muestreos mediante buceo autónomo en el área de } \\
\text { estudio (censos visuales de abundancia). Informe } \\
\text { de la Universidad de La Laguna y la Viceconsejería } \\
\text { de Pesca del Gobierno de Canarias en } 2005 \text {. } \\
\text { - P/B=Z=F+M donde F=C/B y M calculada a partir } \\
\text { de la ecuación empírica de Pauly (1980). Lo y K } \\
\text { de Villamil et al. (2001) } \\
\text { - Ecuación empírica de Pauly et al. (1990). Wo de } \\
\text { Villamil et al. (2001) } \\
\text { - Asunción (Christensen } \text { et al. 2008) } \\
\text { - Havelange } \text { et al. (1997) } \\
\text { - Calculadas a partir del monitoreo de la pesca ar- } \\
\text { tesanal de La Restinga (2003-2005) (Martín-Sosa } \\
\text { et al. 2010). }\end{array}$ \\
\hline
\end{tabular}




\begin{tabular}{|c|c|c|}
\hline $\begin{array}{l}\text { 13. Espáridos } \\
\text { 59,40\% Diplodus vulgaris } \\
\text { 22,71\% Diplodus cervinus } \\
\text { 16,45\% Diplodus sargus } \\
\text { 1,42\% Diplodus puntazzo }\end{array}$ & $\begin{array}{l}\mathrm{Q} / \mathrm{B} \\
\mathrm{U} / \mathrm{Q} \\
\text { Dieta }\end{array}$ & 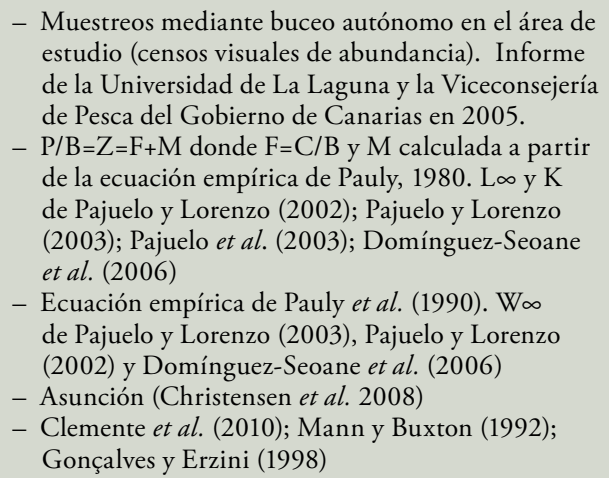 \\
\hline 14. Sparisoma cretense & $\begin{array}{l}\mathrm{Q} / \mathrm{B} \\
\mathrm{U} / \mathrm{Q} \\
\text { Dieta } \\
\text { Descargas }\end{array}$ & $\begin{array}{l}\text { - Muestreos mediante buceo autónomo en el área de } \\
\text { estudio (censos visuales de abundancia). Informe } \\
\text { de la Universidad de La Laguna y la Viceconsejería } \\
\text { de Pesca del Gobierno de Canarias en } 2005 \text {. } \\
\text { - P/B=Z=F+M donde F=C/B y M calculada a partir } \\
\text { de la ecuación empírica de Pauly (1980). L } \infty \text { y K de } \\
\text { González et al. (1993) } \\
\text { - Ecuación empírica de Pauly et al. (1990). Wo de } \\
\text { González et al. (1993) } \\
\text { - Asunción (Christensen et al. 2008) } \\
\text { - Muestreos estomacales de individuos del área de } \\
\text { estudio. } \\
\text { - Calculadas a partir del monitoreo de la pesca ar- } \\
\text { tesanal de La Restinga (2003-2005) (Martín-Sosa } \\
\text { et al. 2010). }\end{array}$ \\
\hline $\begin{array}{l}\text { 15. Peces planctívoros } \\
\text { 67,91\% Boop boops } \\
\text { 30,30\% Chromis limbata } \\
\text { 1,79\% Atheryna presbyter }\end{array}$ & $\begin{array}{l}\mathrm{P} / \mathrm{B} \\
\mathrm{Q} / \mathrm{B} \\
\mathrm{U} / \mathrm{Q} \\
\text { Dieta }\end{array}$ & $\begin{array}{l}\text { - Muestreos mediante buceo autónomo en el área de } \\
\text { estudio (censos visuales de abundancia). Informe } \\
\text { de la Universidad de La Laguna y la Viceconsejería } \\
\text { de Pesca del Gobierno de Canarias en } 2005 \text {. } \\
\text { - P/B=Z=F+M donde F=C/B y M calculada a partir } \\
\text { de la ecuación empírica de Pauly (1980). Lo y K de } \\
\text { Pajuelo y Lorenzo (2000) } \\
\text { - Ecuación empírica de Pauly et al. (1990). Wo de } \\
\text { Morato et al. (2001), Pajuelo y Lorenzo (2000) y } \\
\text { Monteiro et al. (2006) } \\
\text { - Asunción (Christensen et al. 2008) } \\
\text { - Randall (1967) }\end{array}$ \\
\hline $\begin{array}{l}\text { 16. Peces microinvertívoros } \\
\text { 84,26\% Thalassoma pavo } \\
\text { 14,06\% Similiparma lurida } \\
\text { 1,01\% Canthigaster capistrata } \\
0,43 \% \text { Gnatholepis thompsoni } \\
0,16 \% \text { Apogon imberbis } \\
0,08 \% \text { Sphoeroides marmoratus }\end{array}$ & $\begin{array}{l}\mathrm{Q} / \mathrm{B} \\
\mathrm{U} / \mathrm{Q} \\
\text { Dieta }\end{array}$ & $\begin{array}{l}\text { - Muestreos mediante buceo autónomo en el área de } \\
\text { estudio (censos visuales de abundancia). Informe } \\
\text { de la Universidad de La Laguna y la Viceconsejería } \\
\text { de Pesca del Gobierno de Canarias en } 2005 \text {. } \\
\text { - P/B=Z=F+M donde F=C/B y M calculada a partir } \\
\text { de la ecuación empírica de Pauly (1980). L } \infty \text { y K de } \\
\text { Clemente } \text { et al. (2010) } \\
\text { - Ecuación empírica de Pauly et al. (1990). Wo de } \\
\text { Klein (2007) } \\
\text { - Asunción (Christensen et al. 2008) } \\
\text { - Randall (1967); Clemente } \text { et al. (2010); Mance- } \\
\text { ra-Rodríguez y Castro-Hernández (2015); Marna- } \\
\text { ne y Bellwood (2002) }\end{array}$ \\
\hline
\end{tabular}




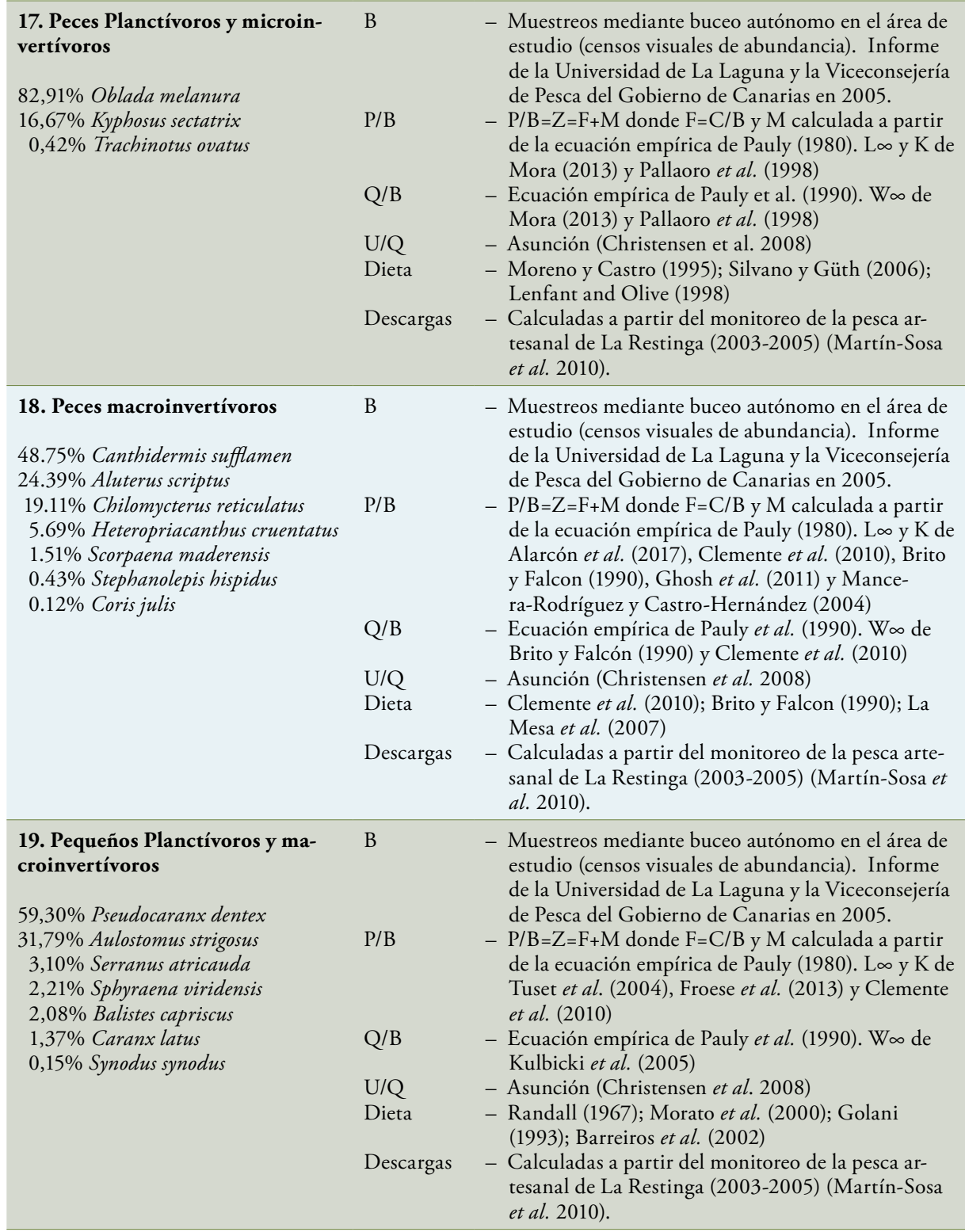




\begin{tabular}{|c|c|c|}
\hline $\begin{array}{l}\text { 20. Peces piscívoros y macroinver- } \\
\text { tívoros } \\
43,31 \% \text { Epinephelus marginatus } \\
40,88 \% \text { Mycteroperca fusca } \\
8,79 \% \text { Seriola dumerili } \\
7,02 \% \text { Bodianus scrofa }\end{array}$ & $\begin{array}{l}\mathrm{P} / \mathrm{B} \\
\mathrm{Q} / \mathrm{B} \\
\mathrm{U} / \mathrm{Q} \\
\text { Dieta } \\
\text { Descargas }\end{array}$ & $\begin{array}{l}\text { - Muestreos mediante buceo autónomo en el área de } \\
\text { estudio (censos visuales de abundancia). Informe } \\
\text { de la Universidad de La Laguna y la Viceconsejería } \\
\text { de Pesca del Gobierno de Canarias en } 2005 \text {. } \\
\text { - P/B=Z=F+M donde F=C/B y M calculada a partir } \\
\text { de la ecuación empírica de Pauly (1980). Lo y K } \\
\text { de Bustos-Leon (2009), Clemente et al. (2010) y } \\
\text { Harmelin (1999) } \\
\text { - Ecuación empírica de Pauly et al. (1990). Wo de } \\
\text { Bustos-Leon (2009) y Ferreira et al. (2008) } \\
\text { - Asunción (Christensen } \text { et al. } 2008) \\
\text { - Linde } \text { et al. (2004), Clemente et al. (2010); Bus- } \\
\text { tos-Leon (2009) } \\
\text { - Calculadas a partir del monitoreo de la pesca ar- } \\
\text { tesanal de La Restinga (2003-2005) (Martín-Sosa } \\
\text { et al. 2010) }\end{array}$ \\
\hline $\begin{array}{l}\text { 21. Morenas } \\
46,03 \% \text { Muraena helena } \\
53,97 \% \text { Gymnotorax unicolor }\end{array}$ & $\begin{array}{l}\mathrm{Q} / \mathrm{B} \\
\mathrm{U} / \mathrm{Q} \\
\text { Dieta } \\
\text { Descargas }\end{array}$ & $\begin{array}{l}\text { - Muestreos mediante buceo autónomo en el área de } \\
\text { estudio (censos visuales de abundancia). Informe } \\
\text { de la Universidad de La Laguna y la Viceconsejería } \\
\text { de Pesca del Gobierno de Canarias en } 2005 \text {. } \\
\text { - P/B=Z=F+M donde F=C/B y M calculada a partir } \\
\text { de la ecuación empírica de Pauly (1980). Lo y K de } \\
\text { Jimenez et al. (2007) } \\
\text { - Ecuación empírica de Pauly et al. (1990). Wo de } \\
\text { Ferreira et al. (2008) } \\
\text { - Asunción (Christensen et al. 2008) } \\
\text { - Matic-Skoko et al. (2010); Randall (1967) } \\
\text { - Calculadas a partir del monitoreo de la pesca ar- } \\
\text { tesanal de La Restinga (2003-2005) (Martín-Sosa } \\
\text { et al. 2010) }\end{array}$ \\
\hline 22. Detritus & B & $\begin{array}{l}\text { - Estimada a partir de la ecuación empírica de Pauly } \\
\text { et al. }(1993) \text { : } \\
\text { Log } \mathrm{D}=0,954 \log \mathrm{PP}+0,863 \log * \mathrm{E}-2,41 \\
\mathrm{E}=40 \text { metros } \\
\mathrm{PP}=\text { Producción primaria (a partir del valor estima- } \\
\text { do de fitoplancton) }\end{array}$ \\
\hline
\end{tabular}




\section{BIBLIOGRAFÍA}

Alarcón, M., Eslava, N., Gonzáles, L.W. y Guevara F. 2017. Growth and mortality of the triggerfish Canthidermis sufflamen (Perciformes: Balistidae) from Los Frailes and Los Testigos Archipelagos, Venezuela. Rev Biol Trop 65(3): 858-867.

Arístegui, J., Hernández-León, S., Montero, M.F. y Gómez, M. 2001. The seasonal planktonic cycle in coastal waters of the Canary Islands. Scient Mar 65, 51-58.

Bode. A., Barquero, S., Varela, M., Braun, J.G. y de Armas, D. 2001. Pelagic bacteria and phytoplankton in oceanic waters near the Canary Islands in summer. Mar Ecol Prog Ser 209: 1-17.

Brey, T. 2001. Population dynamics in benthic invertebrates. A virtual handbook. http://www. awi-bremerhaven.de/Benthic/Ecosystem/FoodWeb/Handbook/main.html. Alfred Wegener Institute for Polar and Marine Research, Germany.

Brito, A. y Falcón, J.M. 1990. Contribución al conocimiento de la distribución y ecología de Chilomycterus atringa (Pises, Diodontidae) en las Islas Canarias. Vieraea 19: 271-275.

Castro, B.G. y Guerra, Á. 1990. The diet of Sepia officinalis (Linnaeus, 1758) and Sepia elegans (D’Orbigny, 1835) (Cephalopoda, Sepioidea) from the Ría de Vigo (NW Spain).

Christensen, V., Walters, C.J., Pauly, D. y Forrest, R. 2008. Ecopath with Ecosim version 6. User guide. Len Oce Fut Project, 235.

Clemente, S., Hernández, J.C., Rodríguez, A. y Brito, A. 2010. Identifying keystone predators and the importance of preserving functional diversity in sublittoral rocky-bottom areas. Mar Ecol Prog Ser 413: 55-67.

Conand, C. 1993. Reproductive biology of the holothurians from the major communities of the New Caledonian Lagoon. Mar Biol 116(3): 439-450.

Couce-Montero, L., Christensen, V. y Castro, J.J. 2015. Effects of small-scale and recreational fisheries on the Gran Canaria ecosystem. Ecol Modell 312: 61-76.

Dalsgaard, J. y Pauly, D. 1997. Preliminary mass-balance model of Prince William Sound, Alaska, for the pre-spill period, 1980-1989. Fisher Cent Res Rep 5(2): 34p.

Domínguez-Seone, R., Pajuelo, J.G., Lorenzo, J.M. y Ramos, A.G. 2006. Age and growth of the sharpsnout seabream Diplodus puntazzo (Cetti, 1777) inhabiting the Canarian archipelago, estimated by reading otoliths and by backcalculation. Fish Res 81(2-3): 142-148.

Eвert, T.A. 1985. Sensitivity of fitness to macroparameter changes: an analysis of survivorship and individual growth in sea urchin life histories. Oecologia 65(4):461-467.

Ferreira, S., Sousa, R., Delgado, J., Carvalho, D. y Chada, T. 2008. Weight-length relationships for demersal fish species caught off the Madeira archipelago (eastern-central Atlantic). J of Appl Ichthyol 24(1): 93-95.

Froese, R., Thorson, J. y Reyes, J.R.B. 2013. A Bayesian approach for estimating length-weight relationships in fishes. J of Appl Ichthyol 2013: 1-7.

Gonçalves, J.M.S. y Erzini, K. 1998. Feeding habits of the two-banded sea bream (Diplodus vulgaris) and the black sea bream (Spondyliosoma gantharus) (Sparidae) from the south-west coast of Portugal. Cybium 22(3): 245-254.

GonzÁlez, J.A. 1993. Sparisoma (Euscarus) cretense (L.) (Pisces, Scaridae): Zoogeografía y ciclo reproductor en las islas Canarias. Courier Forsch.-Inst. Senckenberg 159: 429-435. 
Ghosh, S., Thangavelu, R., Mohamed, G., Dhokia, H.K., Zala, M.S., Savaria, Y.D. y LADANI, A.A. 2011. Sudden emergence of fishery and some aspects of biology and population dynamics of Aluterus monoceros (Linnaeus, 1758) at Veraval. Indian J. Fish 58(1): 31-34.

Golani, D. 1993. The biology of the Red Sea migrant, Saurida undosquamis in the Mediterranean and comparison with the indigenous confamilial Synodus saurus (Teleostei: Synodontidae). Hydrobiologia 271(2): 109-117.

Harmelin. J.G. y Harmelin-Vivien, M. 1999. A review on habitat, diet and growth of the dusky grouper Epinephelus marginatus (Lowe, 1834). Mar Life 9(2): 11-20.

Havelange, S., Lepoint, G., Dauby, P. y Bouquegneau, J.M. 1997. Feeding of the sparid fish Sarpa salpa in a seagrass ecosystem: diet and carbon flux. Mar Ecol 18(4): 289-297.

Hernández, J.C., Toledo, K., Girard, D., Clemente, S., Cubero, E. y Brito, A. 2005. Descripción de la post-larva y primeras fases juveniles de tres equinoideos presentes en las islas Canarias: Diadema antillarum (Philippi, 1845), Paracentrotus lividus (Lamarck, 1816) y Arbaciella elegans (Mortensen, 1910). Vieraea 33: 385-397.

Hernández, C.A. 2016. Sistema de carbono y productividad primaria en zonas costeras de Canarias en un contexto de Cambio Global. Doctoral thesis. Universidad de La Laguna, Tenerife, islas Canarias, 210pp.

Hernández-León, S., Almeida, C., Gómez, M., Torres, S., Montero, I. y Portillo-HahneFELD, A. 2001. Zooplankton biomass and indices of feeding and metabolism in island-generated eddies around Gran Canaria. Jour of Mar Syst 30: 51-66.

Huggett, J., Verheye, H., Escribano, R. y Fairweather, T. 2009. Copepod biomass, size composition and production in the Southern Benguela: Spatio-temporal patterns of variation, and comparison with other eastern boundary upwelling systems. Prog in Oceanography 83: 197-207.

Jiménez, S., Schönhuth, S., Lozano, I.J., González, J.A., Sevilla, R.G., Diez, A. y BauTISTA, J.M. 2007. Morphological, ecological, and molecular analyses separate Muraena augusti from Muraena helena as a valid species. Copeia 2007(1): 101-113.

Kulbicki, M., Guillemot, N. y Amand, M. 2005. A general approach to length-weight relationships for New Caledonian lagoon fishes. Cybium 29(3): 235-252.

Mancera-Rodríguez, N.J. y Castro-Hernández, J.J. 2004. Age and growth of Stephanolepis hispidus (Linnaeus, 1766) (Pisces: Monacanthidae), in the Canary Islands area. Fish Res 66(23): 381-386.

Mann, B.Q. y Buxton, C.D. 1992. Diets of Diplodus sargus capensis and D. cervinus hottentotus (Pisces: Sparidae) on the Tsitsikamma coast, South Africa. Koedoe 35(2): 27-36.

Marnane, M.J. y Bellwood, D.R. 2002. Diet and nocturnal foraging in cardinalfishes (Apogonidae) at one tree reef, great barrier reef, Australia. Mar Ecol Prog Ser 231: 261-268.

Martín-Sosa, P., Cansado, S., Boza, C., González-Lorenzo, J.G., Falcón, J.M. y Villegas, N. 2010. Información pesquera en el entorno de la Reserva Marina de Punta de La Restinga -Mar de Las Calmas- (El Hierro). Periodo 2003-10. IEO, Centro Oceanográfico de Canarias. Informe: 19 pp. + Anexos.

Moens, T. y Vincx, M. 1996. Do meiofauna consume primary production? About many questions and how to answer them. In Integrated Marine System Analysis. European Network for IMS Analysis. FWO Vlaanderen: Minutes of the First Network Meeting (pp. 188-202). 
Monteiro, P., Bentes, L., Coelho, R., Correia, C., Gonçalves, J.M.S., Lino, P.G. y Erzini, K. 2006. Age and growth, mortality, reproduction and relative yield per recruit of the bogue, Boops boops Linné, 1758 (Sparidae), from the Algarve (south of Portugal) longline fishery. J of Appl Ichthyol 22(5): 345-352.

Mora, N. 2013. Crecimiento y aspectos reproductivos del "chub" Kyphosus incisor y Kyphosus sectatrix (Pisces: Kyphosidae) en el PNN Old Providence McBean Lagoon. Isla de Providencia. Caribe colombiano. Doctoral dissertation, Tesis Biologia, Univ. Valle, Cali.

Morato, T., Santos, R.S. y Andrade, J.P. 2000. Feeding habits, seasonal and ontogenetic diet shift of blacktail comber, Serranus atricauda (Pisces: Serranidae), from the Azores, north-eastern Atlantic. Fish Res 49(1): 51-59.

Morato. T., Afonso, P., Lourinho, P., Barreiros, J.P., Santos, R.S. y Nash, R.D.M. 2001. Length-weight relationships for 21 coastal fish species of the Azores, north-eastern Atlantic. Fish Res 50(3): 297-302.

Moreno, T. y Castro, J.J. 1995. Community structure of the juvenile of coastal pelagic fish species in the Canary Islands waters [Spain]. Scie Mar.

Navarro, P.G., García-SAnZ, S. y Tuya, F. 2013. Patrones de abundancia y talla de Holothuria sanctori, Holothuria mammata y Holothuria arguinensis (Echinodermata: Holoturoidea) en la isla de Gran Canaria, Atlántico oriental. Rev Biol Mar Oceanog 48: 273-284.

La Mesa, G., La Mesa, M. y Tomassetti, P. 2007. Feeding habits of the Madeira rockfish Scorpaena maderensis from central Mediterranean Sea. Mar Biol 15(6): 1313-1320.

Linde, M., Grau, A.M., Riera, F. y Massutí-Pascual, E. 2004. Analysis of trophic ontogeny in Epinephelus marginatus (Serranidae). Cybium 28(1): 27-35.

López, J.H. 2000. Biología, ecología y pesca del pulpo común ("Octopus vulgaris», Cuvier 1797) en aguas de Gran Canaria. Doctoral dissertation, Universidad de Las Palmas de Gran Canaria.

Opıtz, S. 1996. Trophic interactions in Caribbean coral reefs (vol. 1085). WorldFish.

Ortega, L., Tuya, F. y Harou, R.J. 2009. El erizo de mar Diadema antillarum (Phillipi, 1845) influye sobre la diversidad y composición de la comunidad de mega-invertebrados vágiles en fondos rocosos del Archipiélago Canario. Rev Biol Mar Oceanog 44: 489-495.

Ortega, I., Martín, A. y Díaz, Y. 2011. Distribución, parámetros poblacionales y dieta de Astropecten marginatus (Asteroidea: Astropectinidae) en el Atlántico venezolano. Rev Biol Trop 59(1): 53-69.

Pajuelo, J.G. y Lorenzo, J.M. 2000. Biology of the sand smelt, Atherina presbyter (Teleostei: Atherinidae), off the Canary Islands (central-east Atlantic). Envir biology of fish 59(1): 91-97.

Pajuelo, J.G. y Lorenzo, J.M. 2002. Age and growth of the annular seabream, Diplodus annularis (Pisces: Sparidae), from the Canarian archipelago (central-east Atlantic). Cienc Mar 28(1): 1-11.

Pajuelo, J.G. y Lorenzo, J.M. 2003. The growth of the common two-banded seabream, Diplodus vulgaris (Teleostei, Sparidae), in Canarian waters, estimated by reading otoliths and by back-calculation. J Appl Ichthyol 19(2): 79-83.

PAuly, D. 1980. On the interrelationships between natural mortality, growth parameters, and mean environmental temperature in 175 fish stocks. ICES Jour of Mar Scie 39: 175-192.

Pauly, D., Christensen, V. y Sambilay, J.V. 1990. Some features of fish food consumption estimates used by ecosystem modelers. ICES. 
Pauly, D., Soriano-Bartz, M. y Palomares, M.L. 1993. Improved construction, parameterization and interpretation of steady-state ecosystem models. In: Christensen, V., Pauly, D. (eds.) Trophic Models of Aquatic Ecosystems.

Ramsay, K., Kaiser, M.J. y Hughes, R.N. 1996. Changes in hermit crab feeding patterns in response to trawling disturbance. Mar Ecol Prog Ser 144: 63-72.

Randall, J.E. 1967. Food habits of reef fishes of the West Indies. Institute of Marine Sciences, University of Miami.

Robinson, L.A., Greenstreet, S.P.R., Reiss, H., Callaway, R., Craeymeersch, J.A.M. y De Boors, I. 2010. Length-weight relationships of 216 North Sea benthic invertebrates and fish. JMBA UK 90(1): 95-104.

Sciberras, M. y Schembri, P.J. 2008. Biology and interspecific interactions of the alien crab Percnon gibbesi in the Maltese Islands. Mar Biol Res 4: 321-332.

Sherr, B.F. y Sherr, E.B. 1984. Role of heterotrophic protozoa in carbon and energy flow in aquatic ecosystem, MJ. Curr perspect in microb ecol 412-423.

Silvano, R.A.M. y Güth, A.Z. 2006. Diet and feeding behavior of Kyphosus spp. (Kyphosidae) in a Brazilian subtropical reef. Braz Arch Biol Technol 49(4): 623-629.

Pallaoro, A., Cetinić, P., Dulčić, J., Jardas, I. y Kraljević, M. 1998. Biological parameters of the saddlead bream Oblada melanura in the eastern Adriatic. Fish Res 38(2): 199-205.

Tuset, V.M., González, J.A., Lozano, I.J. y García-Díaz, M.M. 2004. Age and growth of the blacktail comber, Serranus atricauda (Serranidae), off the Canary Islands (central-eastern Atlantic). Bull of Mar Scie 74(1): 53-68.

Tuya, F., Martín, J.A., Reuss, G.M. y Luque, A. 2001. Food preferences of the sea urchin Diadema antillarum in Gran Canaria (Canary Islands, central-east Atlantic Ocean). JMBA UK 81(5): 845-849.

Tuya, F. y Haroun, R.J. 2006. Spatial patterns and response to wave exposure of shallow water algal assemblages across the Canarian Archipelago: a multi-scaled approach. Mar Ecol Prog Ser 311: 15-28.

Villamil, M.M., Lorenzo, J.M., Pajuelo, J.G., Ramos, A. y Coca, J. 2002. Aspects of the life history of the salema, Sarpa salpa (Pisces, Sparidae), off the Canarian Archipelago (central-east Atlantic). Envir Biology of Fish 63(2): 183-192. 